\title{
An Effective Contour Detection based Image Retrieval using Multi-Fusion Method and Neural Network
}

\section{Rohit Raja}

Guru Ghasidas Vishwavidyalaya: Guru Ghasidas University

Sandeep Kumar ( $\nabla$ er.sandeepsahratia@gmail.com )

Sreyas Institute of Engineering and Technology https://orcid.org/0000-0002-4752-7884

Shilpa Choudhary

Neil Gogte Institute of Technology

Hemlata Dalmia

Sreyas Institute of Engineering and Technology

\section{Research Article}

Keywords: YCbCr, HSV Histogram, Contour, CBIR, Sobel Edge Detection, NN

Posted Date: June 1st, 2021

DOl: https://doi.org/10.21203/rs.3.rs-458104/v1

License: (9) This work is licensed under a Creative Commons Attribution 4.0 International License. Read Full License 


\title{
An Effective Contour Detection based Image Retrieval using Multi-Fusion Method and Neural Network
}

\author{
${ }^{1}$ Rohit Raja, Associate Professor Department of Information Technology, Guru Ghasidas Vishwavidyalaya (A \\ Central University), Bilaspur, Chhattisgarh- 495009 \\ \{drrohitraja1982@gmail.com\} \\ ${ }^{2}$ Sandeep Kumar, Professor ECE Department, Sreyas Institute of Engineering \& Technology, Hyderabad \\ \{er.sandeepsahratia@gmail.com\} \\ ${ }^{3}$ Shilpa Choudhary, Assistant Professor CSE Department, Neil Gogte Institute of Technology, Hyderabad \\ \{shilpachoudhary1987@gmail.com\} \\ ${ }^{4}$ Hemlata Dalmia, Professor ECE Department, Sreyas Institute of Engineering \& Technology, Hyderabad \\ \{dalmiahemlata@gmail.com\}
}

\begin{abstract}
:
Day by day, rapidly increasing the number of images on digital platforms and digital image databases has increased. Generally, the user requires image retrieval and it is a challenging task to search effectively from the enormous database. Mainly content-based image retrieval (CBIR) algorithm considered the visual image feature such as color, texture, shape, etc. The non-visual features also play a significant role in image retrieval, mainly in the security concern and selection of image features is an essential issue in CBIR. Performance is one of the challenging tasks in image retrieval, according to current CBIR studies. To overcome this gap, the new method used for CBIR using histogram of gradient (HOG), dominant color descriptor (DCD) \& hue moment (HM) features. This work uses color features and shapes texture in-depth for CBIR. HOG is used to extract texture features. DCD on RGB and HSV are used to improve efficiency and computation. A neural network (NN) is used to extract the image features, which improves the computation using the Corel dataset. The experimental results evaluated on various standard benchmarks Corel-1k, Corel-5k datasets, and outcomes of the proposed work illustrate that the proposed CBIR is efficient for other state-of-the-art image retrieval methods. Intensive analysis of the proposed work proved that the proposed work has better precision, recall, accuracy.
\end{abstract}

Keywords - YCbCr, HSV Histogram, Contour, CBIR, Sobel Edge Detection, NN.

\section{INTRODUCTION}

The growth of internet usage and the need for high storage for multimedia data produce complexity on image retrieval schemes from large databases. Day by day, multimedia resources increase in numbers because of less cost of most digital devices and advancement in technology. A digital image is always provided an enormous amount of helpful information which could be helpful for 
human. Hence, there is always a need for an effective and efficient image retrieval-based method to retrieve valuable images from the database according to the user's query. Giving a textbase query to the database is a traditional method and Conventional text-based search requires manual annotation whenever a user gives a keyword to search the desired image. The given keyword is verified with the annotation and the particular image is retrieved from the database. However, this methodology is not efficient for extensive image databases due to human perception. So, it will be incomplete [4, 5]. A new method of image retrieval is introduced and it is referred to as CBIR (content-based image retrieval) to overcome the problem of the textbase user query. These methods work on the primary features of the image, such as color shape and texture. These features are the input to the CBIR system. However, these primary features are not able to give the same information as human visual perception gives. Because a human needs a high level of semantics for discrimination, the images and machine will use a low level of primary features. To fill these semantic gap researchers proposed machine learning techniques which can add more semantic meaning. SBIR (SemanticBased Image Retrieval Systems) is a method that can add a high-level semantic meaning of the image to the low level of primary features and produce the desired results [23]. SBIR methods are enhanced with the help of vocabulary and deep learning-based methods. A vocabulary-based method works on the cluster of local features extracted using SURF, SIFT, and HOG. The other image retrieval-based method is content-based image retrieval which uses the inherent features of the image. These inherent features are color, shape and texture. Color features are the most dominant feature of the image because these features information remains the same even if transformation and rotation perform on the image. The most popular color descriptor is DCD which is used in a variety of image retrieval applications. There is another feature extraction technique with the name of EHD. EHD is represented by the MPEG-7 standard of edge-based feature extraction scheme used to illustrate the image geometry. Both Global and local EHDs are utilized to extract the shape of a given image. Further, EHD describes the edge orientation of all sub-images. Different features are extracted from images and CBIR is used to extract images from any database based on various features. Considering the same, this paper combines HOG, DCD and EHD feature extraction techniques to find the color, shape and texture of the image [24-25]. First, CLAP and Sobel technique is applied to find the edges of the images and results combined and the input for the second step. The second step features are extracted from the image using the above feature extraction technique and these features are fed into the neural network for classification purposes. A detailed description is given in Section 3. Further paper is organized as follows: Section 2 gives the existing work details; Section 3 describes the detailed description of the proposed work. Section 4 explains results and comparative analysis and finally, section 5 represents the conclusion and future scope. 


\section{LITERATURE SURVEY}

Guangyi Xie et al. [22], 2020 proposed a novel color descriptor and hu moments-based CBIR method for image retrieval. The proposed work extracted the features and calculated the color descriptor feature for translational, rotational, and image shapes. Finally, the proposed work used three benchmark datasets, Corel-1k, Corel-5k and Corel-10k and achieved superior results compared to other state-of-the-art methods. Ahmad Raza et al. [23], 2018 described a visual-based texton concept for image retrieval. This proposed work provides the correlation between texture, shapes, intensity, color and orientation of an image with the help of a co-occurrence matrix. Finally, the proposed work used three benchmark datasets, Corel-1k, Corel-5k and Corel-10k. The proposed work outperformed compared other methods. Another CBIR algorithm is presented by Atif et al. [18], 2018, for fusing texture and color features. The matching of similarities and feature vectors is performed by Manhattan distance and it uses Corel 1-k data set. The accuracy of the CBIR Algorithm examines the precision and recall and performance of experimental results with other related image retrieval system methods. Yati et al. [15], 2017 proposed an edge-based image retrieval system using RLBP and discrete DWT by reducing dimension. Edge features improve the detection quality. This method achieves $88.37 \%$ on precision. Swati et al. [16], 2014 combine color and texture features for $\mathrm{CBIR}$. $\mathrm{Y}$-matrix of $\mathrm{YCbCr}$ is prepared from extracted edge features that are used in canny edge detection. The distribution of colors is described by computing global statistical description using RGB histogram. Further, a similarity measure is performed using Manhattan distance. The result shows proposed algorithm is better from query image alterations. Dong et al. [19], 2013 has proposed an improved algorithm for image retrieval. This algorithm uses a Gaussian filter and it calculates the preserving of edge information. The Canny edge detection system improves the performance of image retrieval operations.

Table 1: Literature study on existing methodology

\begin{tabular}{|c|c|c|c|c|}
\hline Ref. & $\begin{array}{c}\text { Author \& } \\
\text { Year }\end{array}$ & Methodology & Dataset & Performance \\
\hline$[1]$ & $\begin{array}{l}\text { Guangyi Xie et } \\
\text { al. [22], } 2020\end{array}$ & $\begin{array}{c}\text { Dominant }+\mathrm{Hu} \text { Color } \\
\text { Descriptor and Distance } \\
\text { Calculation }\end{array}$ & $\begin{array}{c}\text { Corel-1k } \\
\text { Corel-5k } \\
\text { Corel-10k }\end{array}$ & $\begin{array}{l}\text { Precision }=78.75 \% \\
\text { Recall }=9.45 \% \\
\text { Precision }=60.05 \% \\
\text { Recall }=7.26 \% \\
\text { Precision }=53.17 \% \\
\text { Recall }=6.38 \%\end{array}$ \\
\hline$[2]$ & $\begin{array}{l}\text { Ahmad Raza et } \\
\text { al. [23], } 2018\end{array}$ & CPV-THF & $\begin{array}{c}\text { Corel-1k } \\
\text { Corel-5k } \\
\text { Corel-10k }\end{array}$ & $\begin{array}{l}\text { Precision }=80.79 \% \\
\text { Recall }=9.69 \% \\
\text { Precision }=63.90 \% \\
\text { Recall }=7.66 \%\end{array}$ \\
\hline
\end{tabular}




\begin{tabular}{|c|c|c|c|c|}
\hline & & & & $\begin{array}{l}\text { Precision }=52.28 \% \\
\text { Recall }=6.27 \%\end{array}$ \\
\hline$[3]$ & $\begin{array}{c}\text { Naushad } \\
\text { Varish et al. } \\
{[24], 2020}\end{array}$ & ATT & $\begin{array}{l}\text { Corel-1k } \\
\text { Corel-10k }\end{array}$ & $\begin{array}{l}\text { Precision }=89.00 \% \\
\text { Recall }=13.50 \% \\
\text { F-Score }=29.49 \% \\
\text { Precision }=90.00 \% \\
\text { Recall }=2.20 \% \\
\text { F-Score }=6.92 \%\end{array}$ \\
\hline$[4]$ & $\begin{array}{l}\text { Ali Ahmed et } \\
\text { al. [25], } 2020\end{array}$ & $\mathrm{HSV}+\mathrm{GLCM}$ & $\begin{array}{l}\text { Kvasir Dataset } \\
\text { PH2 Dataset }\end{array}$ & $\begin{array}{l}\text { Precision }=95.8 \% \\
\text { Precision }=80.7 \%\end{array}$ \\
\hline$[5]$ & $\begin{array}{l}\text { Ali Ahmed } \\
{[26], 2020}\end{array}$ & RFRM + GLCM & Kvasir Dataset & Precision $=86.25 \%$ \\
\hline$[6]$ & $\begin{array}{l}\text { Kenta Iida et al. } \\
\text { [27], } 2020\end{array}$ & SCD + CEDD & $\begin{array}{l}\text { Ukbench } \\
\text { Dataset }\end{array}$ & $\mathrm{mAP}=94.19 \%$ \\
\hline$[7]$ & $\begin{array}{c}\text { Jingyu Wang } \\
\text { et al. [28], } \\
2018 \\
\end{array}$ & $\begin{array}{c}\text { Contour Extraction + MLGF } \\
+ \text { GF-HOG }\end{array}$ & Coco Dataset & Time $=1-2 \mathrm{~s}$ \\
\hline$[8]$ & $\begin{array}{l}\text { Famao Ye et } \\
\text { al. [29], } 2019\end{array}$ & $\mathrm{~K}-\mathrm{NN}+\mathrm{CNN}$ & $\begin{array}{c}\text { UCMD and } \\
\text { Pattern Net } \\
\text { databases }\end{array}$ & \\
\hline$[9]$ & $\begin{array}{l}\text { Xiaoqing Li et } \\
\text { al. [30], } 2020\end{array}$ & CNN-SURF + RANSAC & $\begin{array}{l}\text { Oxford5k } \\
\text { UKB }\end{array}$ & $\begin{array}{l}\text { Map }=88.3 \% \\
\text { N-S score }=3.92\end{array}$ \\
\hline$[10]$ & $\begin{array}{c}\text { Mudhafar Jalil } \\
\text { Jassim Ghrabat } \\
\text { et al. [31], } \\
\text { 2019 }\end{array}$ & $\begin{array}{c}\text { Multiple Ant Colony } \\
\text { Optimization + GDBM }\end{array}$ & $\begin{array}{c}\text { European-1M, } \\
\text { Flickr } \\
\text { Corel 1K } \\
\text { LFW }\end{array}$ & $\begin{array}{l}\text { Recall }=31.9 \% \\
\text { Precision }=90.71 \% \\
\mathrm{mAP}=85.4 \% \\
\text { Accuracy }=98.92 \%\end{array}$ \\
\hline & \multicolumn{4}{|c|}{$\begin{array}{l}\text { ATT }=\text { Adaptive Tetrolet Transform, CPV-THF }=\text { Correlated Primary Visual }+ \text { Texton } \\
\text { Histogram Features, GLCM }=\text { Grey-Level Co-Occurrence Matrix, RFRM }=\text { Relevance } \\
\text { Feedback Retrieval Method, SCD }=\text { Scalable Color Descriptor, CEDD }=\text { Color and Edge } \\
\text { Directivity Descriptor, MLGF }=\text { Multiscale Local Gradient Features, K-NN = k- } \\
\text { Nearest Neighbor, CNN = Convolutional Neural Network, SURF }=\text { Speeded Up Robust } \\
\text { Features, RANSAC = RANdom SAmple Consensus, GDBM = Greedy Learning Deep } \\
\text { Boltzmann Machine Classifier }\end{array}$} \\
\hline
\end{tabular}

\section{Proposed WoRK}

This research has proposed an arrangement of texture, color, and shape features. This system is to achieve better precision and reduce recall rate as evaluated to existing work. This algorithm consists of the following stages: (a) Pre- Processing (b) Feature Extraction (c) Classification as shown in Fig. 1. 


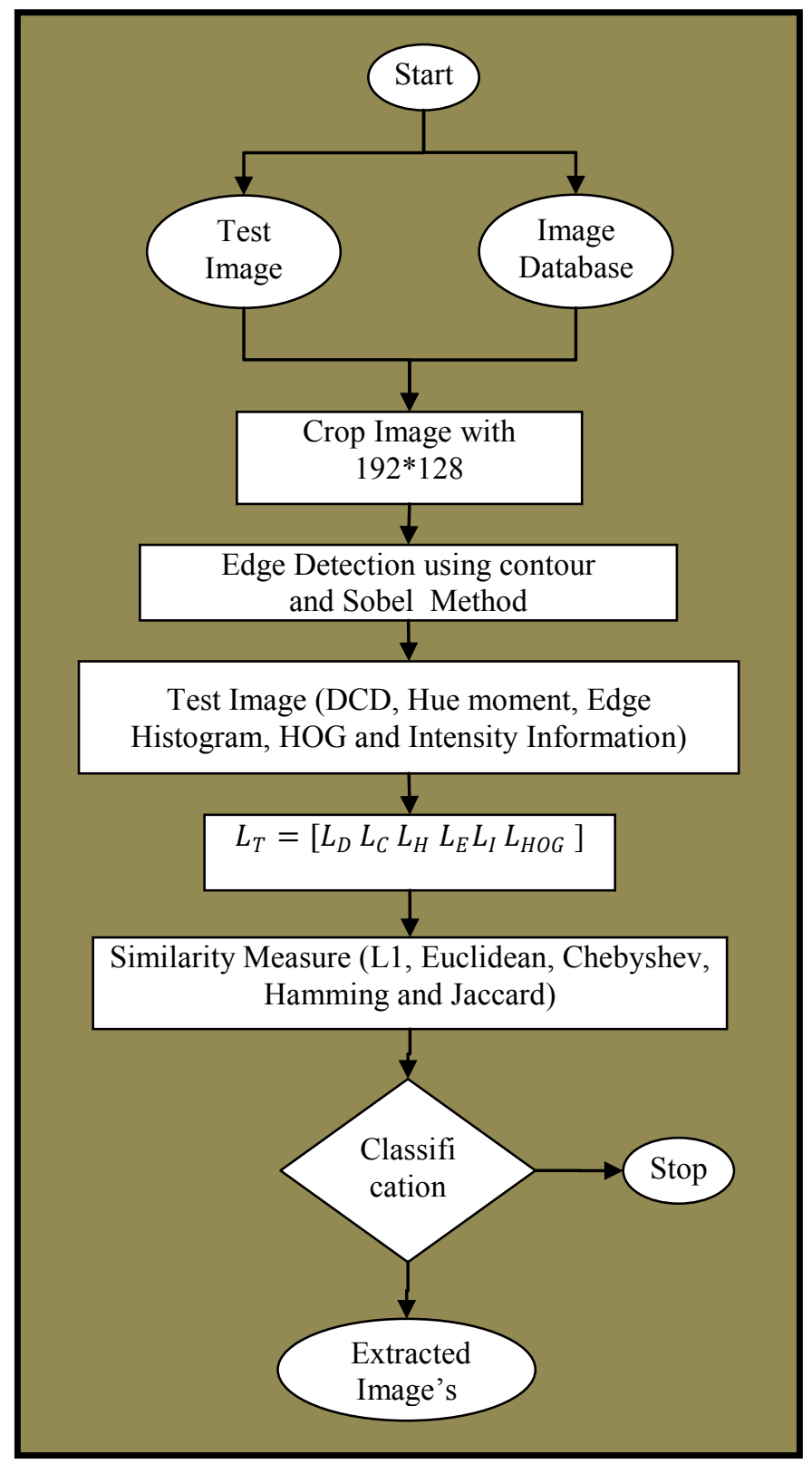

Fig. 1. Flow Chart of Proposed Work

Step-1: Image Acquisition: It is a method of acquiring the digital image from some resources. In this step standard datasets are acquired and given as an input for the next step.

Step-2: Pre Processing: Resizing of image is a preprocessing technique used to change input image size. In the proposed method, all the images are converted into $192 * 128$ sizes.

Step-3: ROI Segmentation: Contour and Sobel edge detector are used to segment the object from the resized image shown in Fig. 2 \& 3. In this step, image is divided into $\mathrm{H}, \mathrm{S}$, and $\mathrm{V}$ components and then bin of size 8 to each component. An edge detection technique is helpful in preserving the shape of the image and also identifies the edges of the image. First RGB image is converted into HSV. First Sobel and CLAP based contour algorithm is applied on the H and V 
components of the image and. Now the detected edges results are combined with unmodified S component. The procedure is shown in Fig. 2.

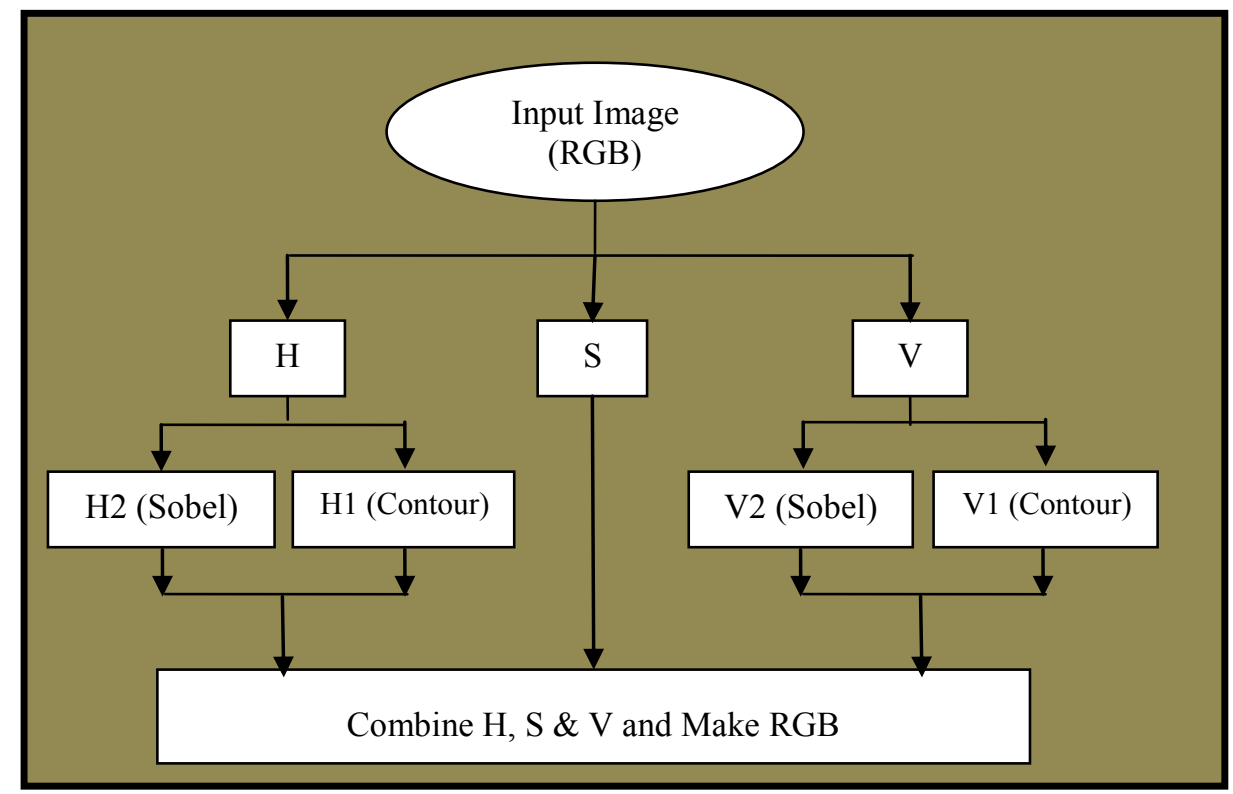

Fig. 2. Flow Chart of edge detection of the proposed method.

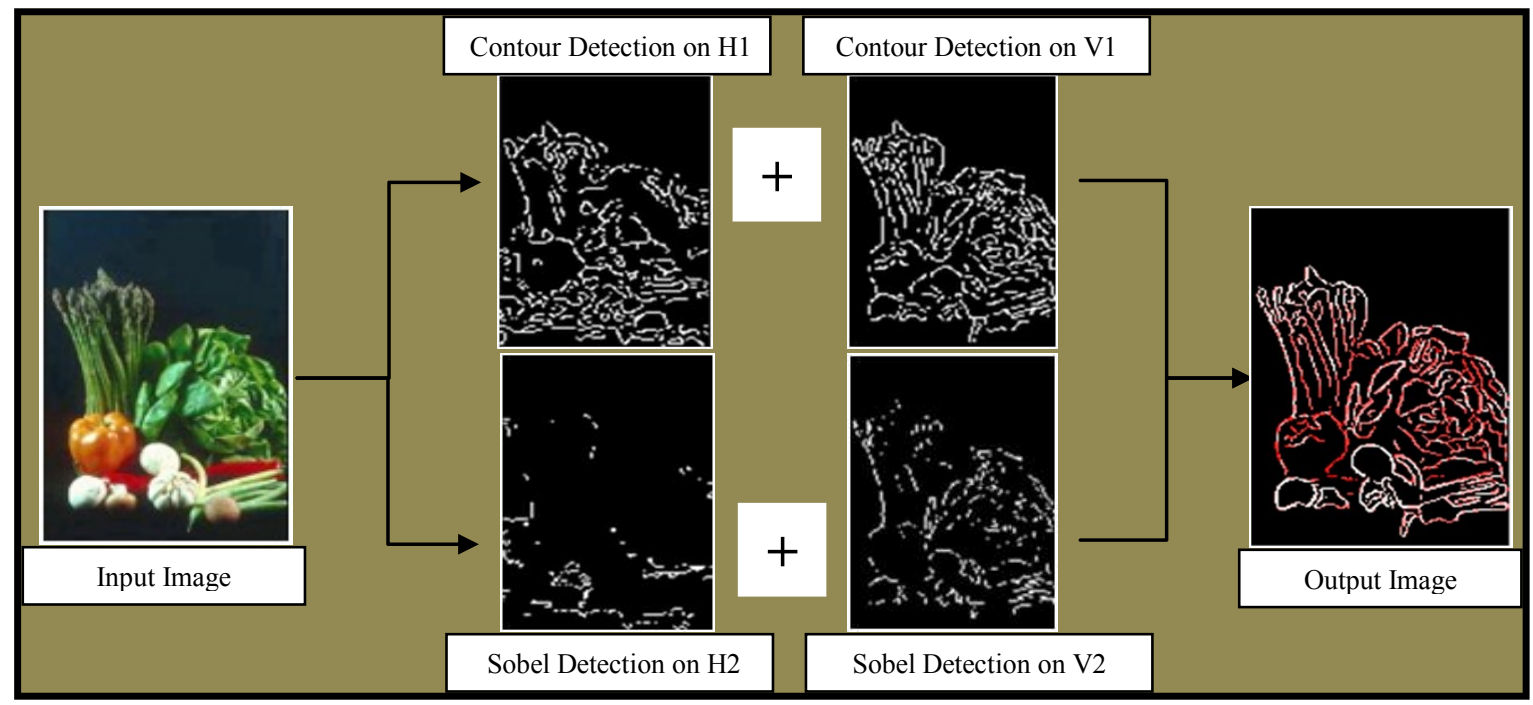

Fig. 3. Results on Vegitables Image .

\section{- Sobel Edge Operator}

The mask of the edge Sobel operator is defined by horizontal $\left(G_{x}\right)$ and vertical direction $\left(G_{y}\right)$ components as shown in equations (1) and (2), respectively.

$$
G_{x}=\left[\begin{array}{ccc}
-1 & -2 & -1 \\
0 & 0 & 0 \\
1 & 2 & 1
\end{array}\right]
$$




\section{- CLAP based wireframe model}

$$
G_{y}=\left[\begin{array}{lll}
-1 & 0 & 1 \\
-2 & 0 & 2 \\
-1 & 0 & 1
\end{array}\right]
$$

The contour or wireframe of the object is a simple curve that joins all the points of the same intensity. The CLAP-based wireframe model[22] is used to identify the contour/wireframe of the input image. This approach traces the whole image and if all the boundary points have the same values, then it will detect that part of the image and this process will continue until it finds all the edge of the given input image. It can find all the curves, lines and junctions.

\section{Step-4: Feature Extraction using CBIR}

It is a process of automatically determining the compressed and meaningful representation of the image. Various feature extraction techniques give better results even though every method has its own pros and cons. Therefore, In this paper, feature extraction has been done in three different methods and then results are combined for better accuracy. Further, a brief explanation of feature extraction methods is discussed.

\section{- Histogram Oriented Gradient (HOG)}

Object recognition and facial detection techniques use gradient-oriented histogram (HOG) as descriptors [6]. HOG analyses the object and shape by examining either the direction of edges or examining the gradient distribution of the density. The HOG separates an image into different cells and then calculates the gradient-directed histogram for each cell. The following three steps can accomplish this.

First Step: Deriving masks of both horizontal M1 and vertical M2 is illustrated in the following equations. These masks are used for determining the gradient of image points.

$$
M_{1}=\left[\begin{array}{lll}
-1, & 0, & 1
\end{array}\right] M_{2}=\left[\begin{array}{lll}
-1, & 0, & 1
\end{array}\right]^{T}
$$

Generally, the gradient's supreme assessment will matter because of a contrast between the white background of a black object and the black background of a white object. Both of these two regions have the same response.

Second Step: The orientation is calculated at first, then measurement of gradients is performed in the image and which is treated as the angle:

$$
\theta=\arctan \frac{g_{x}}{g_{y}} \text { classically } \in[0, \pi]
$$


Third Step: The image is split into several cells to construct the histogram of an image. The construction of the histogram measures the gradients bar for the particular orientation interval for each cell.

\section{- Dominant Color Descriptor}

MPEG-7is widely used dominant color descriptor (DCD) and which is highly used in image retrieval scheme. DCD represents features of an image and color distributions by using an intuitive, compact and practical format. Further, the DCD is utilized for the image retrieval scheme based on constant numbers MPGA-7 format and describes the color and other more minor features. The main contribution is reducing time on image retrieval and increasing the performance. The DCD is structured as F, as shown in equation (7).

$$
\mathrm{F}=\{\text { pi, ci, vi, } \mathrm{s}\}, 1,2 \ldots \ldots \mathrm{NDCD}
$$

Where NDCD gives the count of principal or dominant colors, the percentage of the pixels in the image subsequent with the $i^{\text {th }}$ dominant color is provided by pi, vector of $i^{\text {th }}$ dominant color is represented by vi. An operational variation on the dominant color pixels is represented by ci. Spatial coherency is represented by s; usually, it is fixed as o in wide applications [7].

\section{- Edge Histogram Descriptor (EHD)}

EHD is represented by the MPEG-7 standard of edge-based feature extraction scheme used to illustrate the image geometry. Both Global and local EHDs are utilized to extract the shape of a given image. Further, EHD describes the edge orientation of all sub-images [8]. EHD categorizes the orientation of edge in five different classes. They are vertical, horizontal, 45degree, 135-degree and no-direction orientations [9].

i. The calculation of local EHD is performed by splitting the image into 16 sub-images and measuring the orientation of edges in all sub-images [10-11].

ii. The evaluation of global edge distributions on the given image is performed by global EHD. The bin value of global EHD depends on the five types of the orientation of edge pixels. Thus, the local EHD consists of 80 bins and the global EHD consists of 85 bins [12].

\section{STEP-5: NEURAL NETWORK}

The composition of the neural network consists of nodes, connections between nodes, and different processing elements [14]. The connection between any two different nodes consists of 
some weight. This weight is utilized to measure the effect of one node on another. An input node is derived from a unit as a sub-set and some other sub-sets are used as output nodes[15-16]. A value is assigned and the network allows the value to propagate to the output node. The mapping of a neural network is saved in weights on the network. The Feed-forward neural network(FFNN) [17-18] (Figure 1) allows signals to pass through one path only, starting input to the output. No feedback or loop available and thus, the output nodes do not affect the similar layer. The FFNN tends to forward directly with an association of input and output [19].

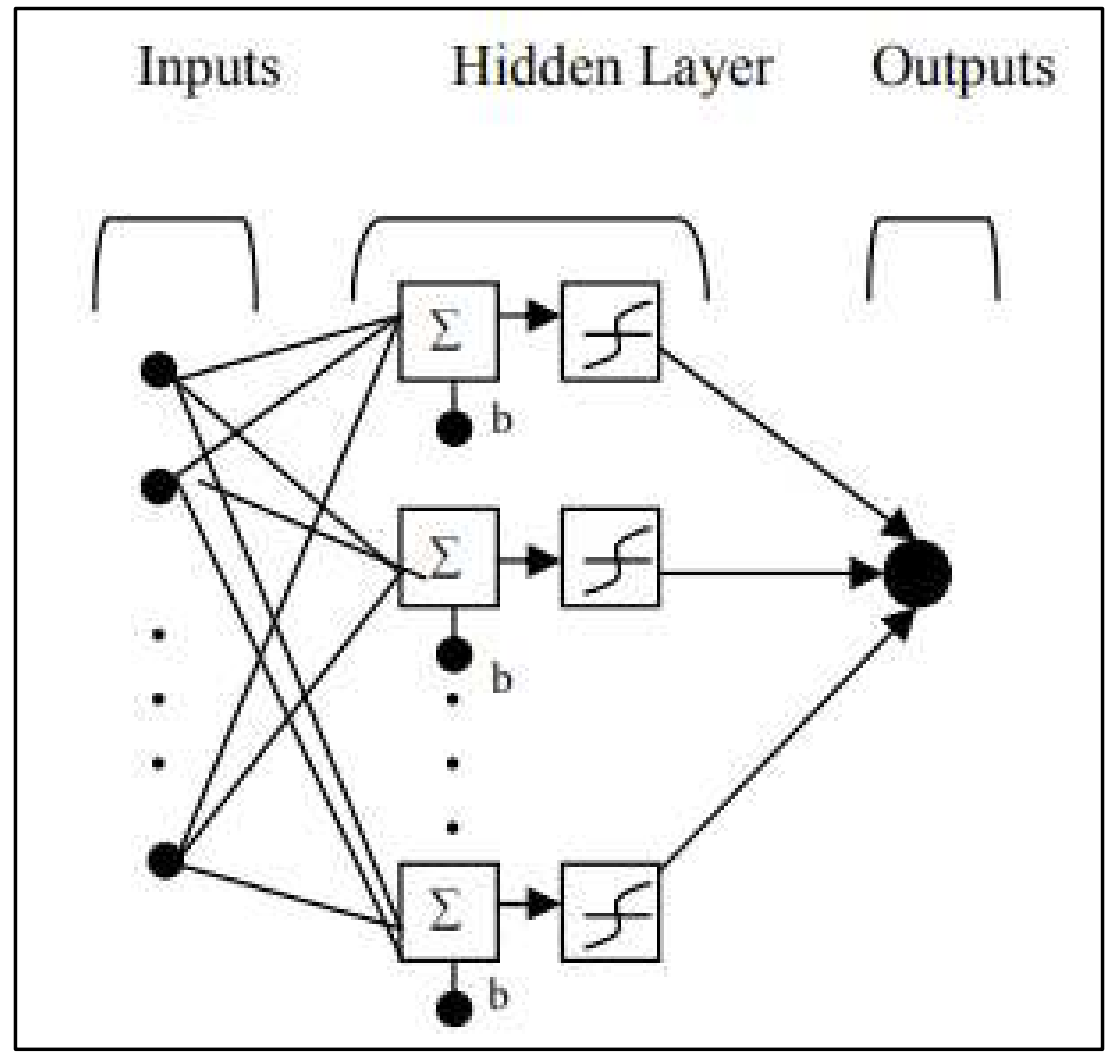

Fig. 4. FEED-Forward Network

In FFNN, only the input and output mapping is performed directly but not aligned with the previous input. However, the next coming scheme call series arranged in a gathering of particular call series and this series cannot be anything[20-21]. For instance, two series like (fstat, old mmap, close) and (execve, uname,brk) are valid series and meaningless due to the requirement of opening a file before making any effort to close. Therefore, a sequence or series similar to (open, fstat, old_mmap) is required. Thus, the series or sequences are connected with the previous series or sequence and interrupt the detection of information required to store. 


\section{Proposed Algorithm}

1. Consider RGB image as a query image $Q(x, y)$ with the size of $\mathrm{M}^{*} \mathrm{~N}$.

2. Resize the image with $192 * 128$ sizes.

3. Convert RGB image into HSV color space.

4. Edge orientation $L_{E}$ is used for edge detection and shape feature extraction on HSV Images.

5. DCD is applied on HSV $L_{D}$ and RGB color space $L_{D 1}$. Find the histogram for three components by Edge Histogram. Generate the filters for the five types of edges:

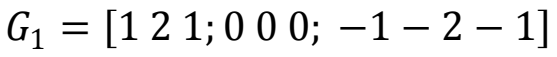

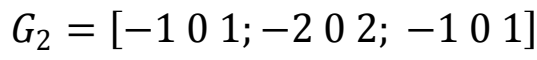

$$
\begin{aligned}
& G_{3}=[22-1 ; 2-1-1 ;-1-1-1] \\
& G_{4}=[-122 ;-1-12 ;-1-1-1]
\end{aligned}
$$

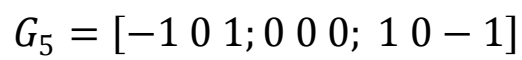

Determine the index of the orientation and maximum Sobel gradient

$$
[m, k]=\max \left(g_{k m}, 3\right)
$$

Where $m$ denotes gradient, $k$ represents the orientation, $g_{k m}$ represents query image using CLAP based wireframe model and finds the edges. Types of orientations are multiplied with edge image:

$$
\mathrm{d}=(\mathrm{k} \times \mathrm{ed})
$$

Where $\mathrm{d}$ denotes detected image, ed denotes edges detected by this method.

$$
\mathrm{L}_{\mathrm{E}}=\operatorname{hist}(\mathrm{d}(:, 5))
$$

Where $L_{E}$ denotes the final histogram of an image in five directions

6. Calculate moment $(\mathrm{p}, \mathrm{q})$ :-

$$
m_{p, q}=\sum_{x=0}^{M-1} \sum_{y=0}^{N-1} f(x, y) x^{p} y^{q}
$$

where $p$ is the order of $x$ and $q$ is the order of $y$

7. A mid moment is mostly the alike as the instants illustrate, excluding that they signify values to replace the standards of $x$ as well as $y$ utilized in the equations

$$
\begin{gathered}
\beta_{p, q}=\sum_{x=0}^{M-1} \sum_{y=0}^{N-1} f(x, y)\left(x-x_{\text {avg }}\right)^{p}\left(y-y_{a v g}\right)^{q} \\
\text { Where } x_{\text {avg }}=\frac{m_{10}}{m_{00}} \text { and } y_{\text {avg }}=\frac{m_{01}}{m_{00}}
\end{gathered}
$$


8. The normalized instant $s \gamma_{p, q}$ is the similar to the mid instants apart from that all divided through the suitable power of $\mathrm{m}_{00}$

$$
\gamma_{p, q}=\frac{\gamma_{p, q}}{m_{00}^{\frac{p+q}{2}+1}}
$$

9. Hu invariant instants are linear groupings of the mid instants and here it is shown that the instants (eight $\mathrm{Hu}$ instants) as:

$$
\begin{aligned}
& I 1=\gamma_{20}+\gamma_{02} \\
& I 2=\left(\gamma_{20}-\gamma_{02}\right)^{\wedge} 2+4 \times \gamma_{11} \\
& I 3=\left(\gamma_{30}-3 \times \gamma_{12}\right)^{\wedge} 2+\left(\gamma_{03}-3 \times \gamma_{21}\right)^{\wedge} 2 \\
& I 4=\left(\gamma_{30}+\gamma_{12}\right)^{\wedge} 2+\left(\gamma_{03}+\gamma_{21}\right)^{\wedge} 2 \\
& I 5=\left(\gamma_{30}-3 \times \gamma_{12}\right) \times\left(\gamma_{30}+\gamma_{12}\right) \times\left(\left(\gamma_{30}+\gamma_{12}\right)^{\wedge} 2-3 \times\left(\gamma_{21}+\gamma_{03}\right)^{\wedge} 2\right)+(3 \\
& \left.\times \gamma_{21}-\gamma_{03}\right) \times\left(\gamma_{21}+\gamma_{03}\right) \times\left(3 \times\left(\gamma_{30}+\gamma_{12}\right)^{\wedge} 2-\left(\gamma_{30}+\gamma_{12}\right)^{\wedge} 2\right) \\
& I 6=\left(\gamma_{20}-\gamma_{02}\right) \times\left(\left(\gamma_{30}+\gamma_{12}\right)^{\wedge} 2-\left(\gamma_{21}+\gamma_{03}\right)^{\wedge} 2\right)+4 \times\left(\gamma_{30}+\gamma_{12}\right) \times\left(\gamma_{21}+\gamma_{03}\right) \\
& I 7=\left(3 \times \gamma_{21}-\gamma_{03}\right) \times\left(\gamma_{30}+\gamma_{12}\right) \times\left(\left(\gamma_{30}+\gamma_{12}\right)^{\wedge} 2-3 \times\left(\gamma_{21}+\gamma_{03}\right)^{\wedge} 2\right)+\left(\gamma_{03}\right. \\
& \left.-3 \times \gamma_{21}\right) \times\left(\gamma_{21}+\gamma_{03}\right) \times\left(3 \times\left(\gamma_{30}+\gamma_{12}\right)^{\wedge} 2-\left(\gamma_{30}+\gamma_{12}\right)^{\wedge} 2\right) \\
& I 8=\left(\gamma_{11} \times\left(\gamma_{30}+\gamma_{12}\right)^{\wedge} 2-\left(\gamma_{30}+\gamma_{12}\right)^{\wedge} 2-\left(\gamma_{20}-\gamma_{02}\right) \times\left(\gamma_{30}+\gamma_{12}\right) \times\left(\gamma_{21}\right.\right. \\
& \left.+\gamma_{03}\right)
\end{aligned}
$$

10. Find HOG descriptor using the below formula:

The gradient magnitude $|G(x, y)|$ and gradient angle $\emptyset(x, y)$ at position $(\mathrm{x}, \mathrm{y})$ are given by

$$
\begin{gathered}
|G(x, y)|=\sqrt{G_{x}(x, y)^{2}+G_{y}(x, y)^{2}} \\
\tan (\varnothing(x, y))=\frac{G_{y}(x, y)}{G_{x}(x, y)}
\end{gathered}
$$

The Gaussian weights of the incline magnitudes contained by a chunk are achieved through multiply there calculated standards of the Gaussian filter matrix stored in a ROM. The magnitude of the weighted gradient is added for every bin to generate a histogram of a cell. After that, the histograms of four cells are concatenated for deriving the resultant block histogram.

11. Step 1 to Step 10 repeats again in the image dataset.

12. Then, by splicing $\left[L_{C} L_{H} L_{E} L_{I}\right]$ combined, the histogram of an image can be denoted through $L_{T}=\left[\begin{array}{lll}L_{C} & L_{H} & L_{E} \\ L_{I}\end{array}\right]$

Like this, $\mathrm{L}_{\mathrm{T}}$ will be 219 measurements rather than 974 measurements, which can spare the calculation time and memory space.

13. Calculate the distance metrics of test image and database through ED, MD, HD, Chebyshev and JD. We have utilized MD, which is the most unsurprising measurement for figuring the absence of association among two vectors. 
Given two vectors $\mathrm{Q}$ and $\mathrm{D}$, where

$$
\mathrm{d}(\mathrm{Q}, \mathrm{D})=\sum_{\mathrm{m}} \sum_{\mathrm{n}}\left|\mathrm{f}_{\mathrm{q}}(\mathrm{m}, \mathrm{n})-\mathrm{f}_{\mathrm{d}}(\mathrm{m}, \mathrm{n})\right|
$$

Where $f_{q}$ speaks to the feature vector of the question picture and $f_{d}$ speaks to include a vector of pictures in the database. The pictures are recovered by the positioning given to them. Pictures with higher comparability will be given higher positions.

14. Using the $\mathrm{NN}$ classifier, classify the images and joined all features.

15. Find accuracy and precision of extracted images.

$$
\begin{gathered}
\text { Precision }=\frac{\text { No. of relevant image extracted }}{\text { Totalnumberofimage extracted. }} \\
\text { Accuracy }=100 \times((\mathrm{TP}+\mathrm{TN}) / \mathrm{N})
\end{gathered}
$$

Where TP presents the truly positive and TN gives truly negative, further $\mathrm{N}$ deals with the size of the given dataset.

\section{RESULT ANALYSIS}

The implementation of the proposed methodology is done on Mat lab version 2014a and the Matlab deep learning toolbox is used for evaluation. The experiments are performed on NVIDIA GPU-based system with 8GB RAM. Corel-1k and the Corel-5k are utilized and 5 fold cross-validation technique is used and $80 \%$ of data is used for training purposes and $20 \%$ of data is used for testing purposes to evaluate the performance of the proposed methodology. A sample of the validation performance is shown in Fig. 5 and the confusion matrix is shown in Fig. 6. Classification is done through a neural network and various hyperparameters have to be set and value of these parameters is shown in Table 2.

Table 2: Details of Neural Network Classifier

\begin{tabular}{|c|c|c|}
\hline Parameter Name & Corel-1K & Corel-5K \\
\hline Epocs & 45 & 85 \\
\hline Learning Rate & 0.001 & 0.001 \\
\hline Dropout & 0.30 & 0.30 \\
\hline Batch Size & 64 & 64 \\
\hline
\end{tabular}




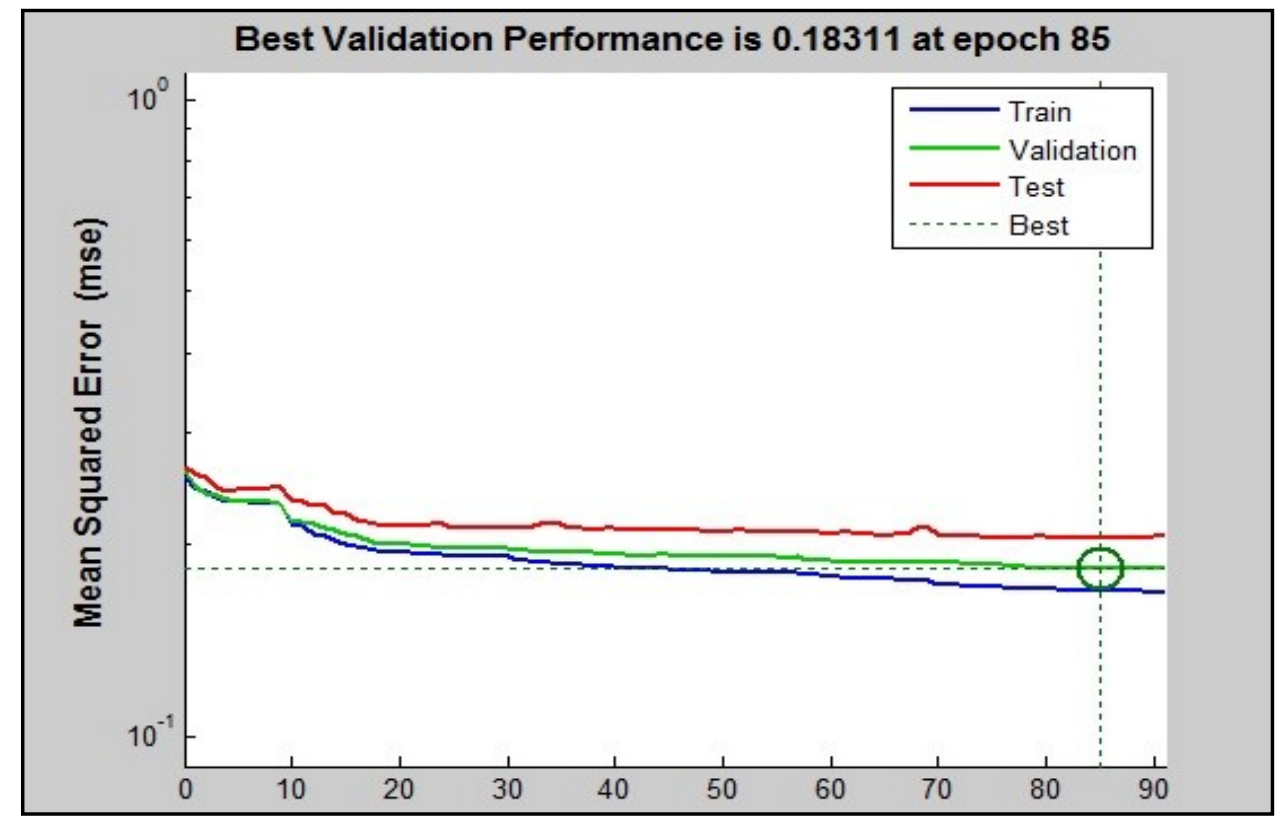

Fig. 5. Validation performance sample

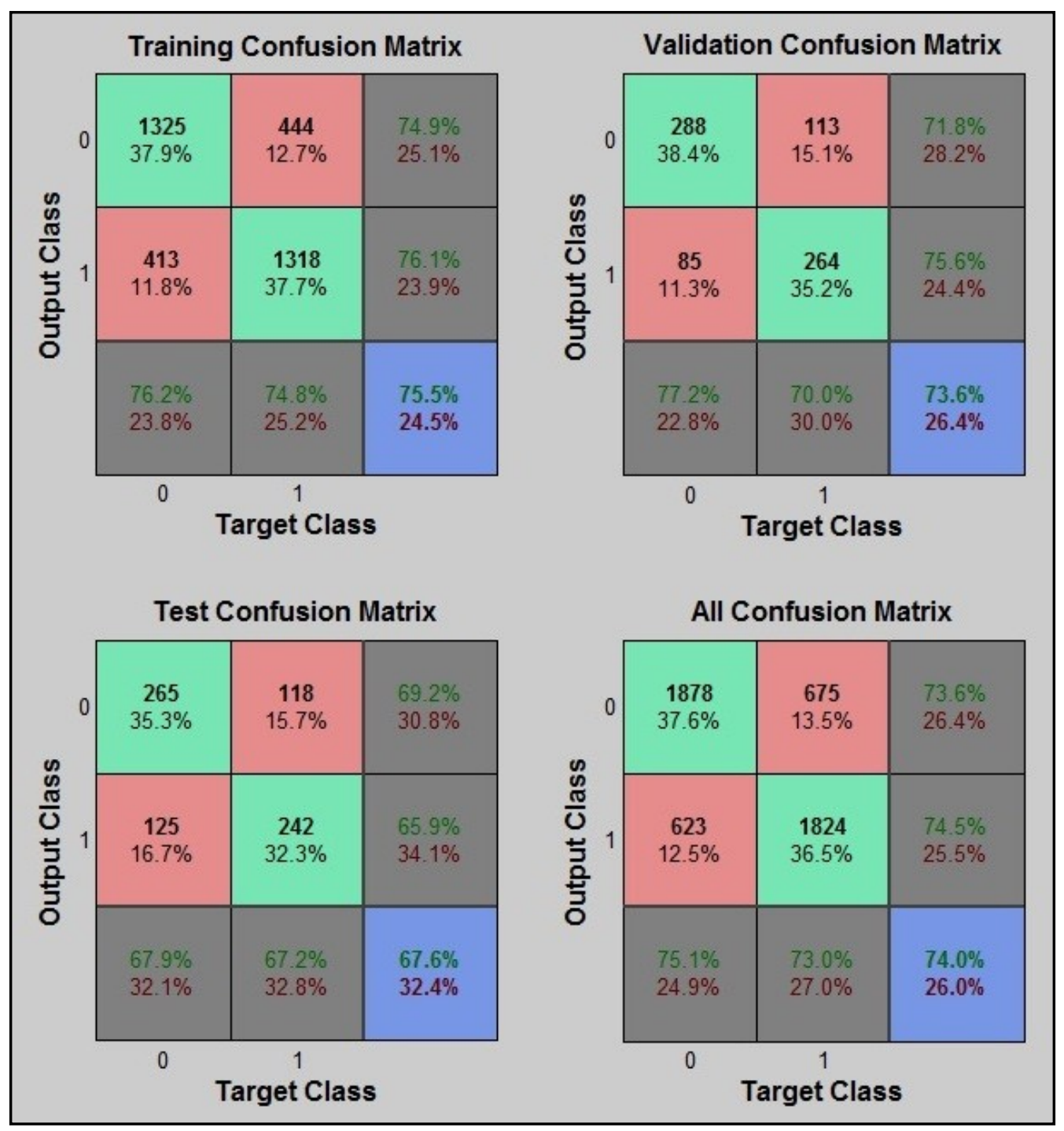

Fig. 6. Overall confusion matrix on benchmark datasets 


\subsection{Evaluation Parameters}

- Accuracy

$$
\text { Accuracy }=\frac{T P+T N}{(T P+T N+F P+F N)}
$$

- Precision

$$
\text { Precision }=\frac{T P}{T P+F P}
$$

- Recall

$$
\text { Recall }=\frac{T P}{T P+F N}
$$

- F1 Score

$$
\text { F1 Score }=2 * \frac{\text { precision } * \text { Recall }}{\text { Precision }+ \text { Recall }}
$$

$\mathbf{T P}=$ True Positive, $\mathbf{T N}=$ True Negative, $\mathbf{F P}=$ False Positive, $\mathbf{F N}=$ False Negative

\subsection{Dataset Used and Results}

- Corel-1k Dataset: This dataset consists of ten different classes of images and for each class, 1000 images are available and few samples are shown in Fig. 7. GUI of the proposed method is created to provide a better experience to the user, as shown in Fig 8. Precision and recall are calculated to evaluate the performance of the proposed method accuracy. Similarity index of the image is calculated using L1, Euclidean, Chebychev, Hamming, Jaccard function and comparative analysis is shown in Table 3 and graph plotting is shown in Fig. 9.

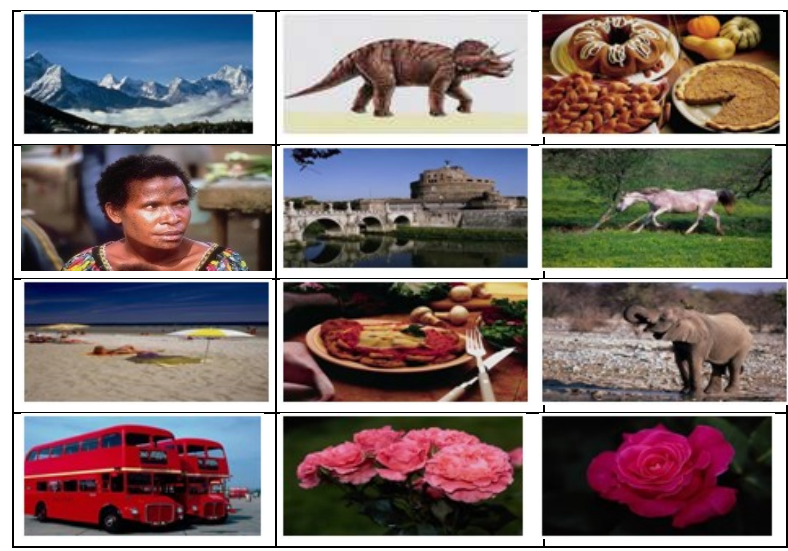

Fig. 7. Corel-1k (Few images from the dataset) 


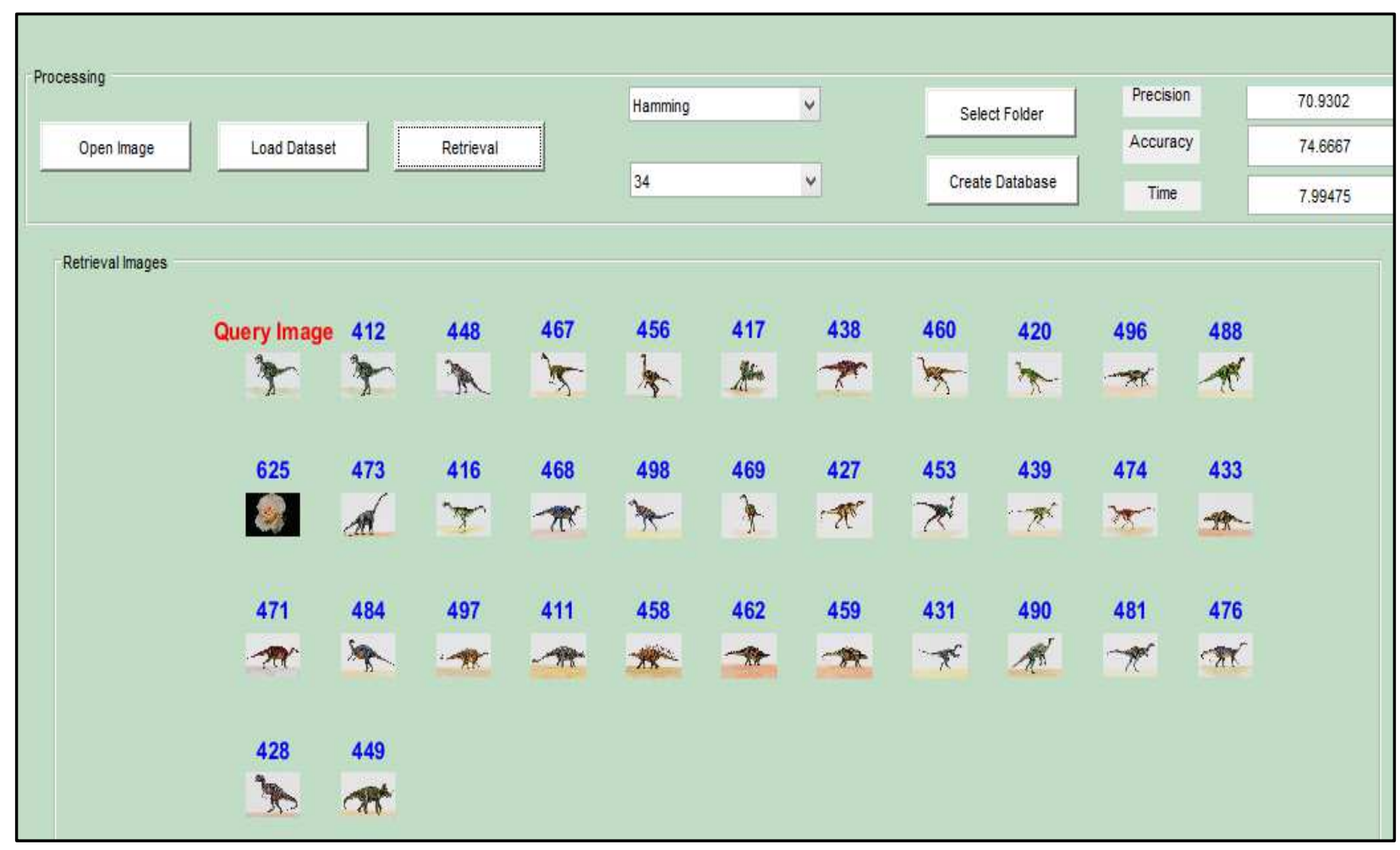

Fig. 8. Dinosaur images are extracted from the dataset against 1 test/query image (Corel-1k dataset).

Table 3: The different similarity metrics based on precision value (L1, Euclidean, Chebychev, Hamming, Jaccard) on Corel-1K Dataset

\begin{tabular}{|c|c|c|c|c|c|}
\hline & L1 & Euclidean & Chebychev & Hamming & Jaccard \\
\hline African & 74.50 & 84.07 & 91.81 & 86.47 & 89.56 \\
\hline Flowers & 86.36 & 94 & 77.92 & 88.51 & 83.07 \\
\hline Horses & 75.38 & 80.90 & 81.42 & 85 & 85.51 \\
\hline Dinosaurs & 82.72 & 88.43 & 89.84 & 94.04 & 77.79 \\
\hline Food & 90 & 81.42 & 81.18 & 94.90 & 91.39 \\
\hline Beach & 89 & 84.3 & 88.4 & 93.2 & 87.3 \\
\hline Buildings & 88.9 & 85.6 & 81 & 91.89 & 89.9 \\
\hline Buses & 92.3 & 87.9 & 93.15 & 89.4 & 91.2 \\
\hline Elephants & 94.17 & 93.2 & 92.01 & 93.76 & 95.02 \\
\hline Mountain & 93.1 & 90.1 & 87.2 & 94.4 & 91.3 \\
\hline
\end{tabular}




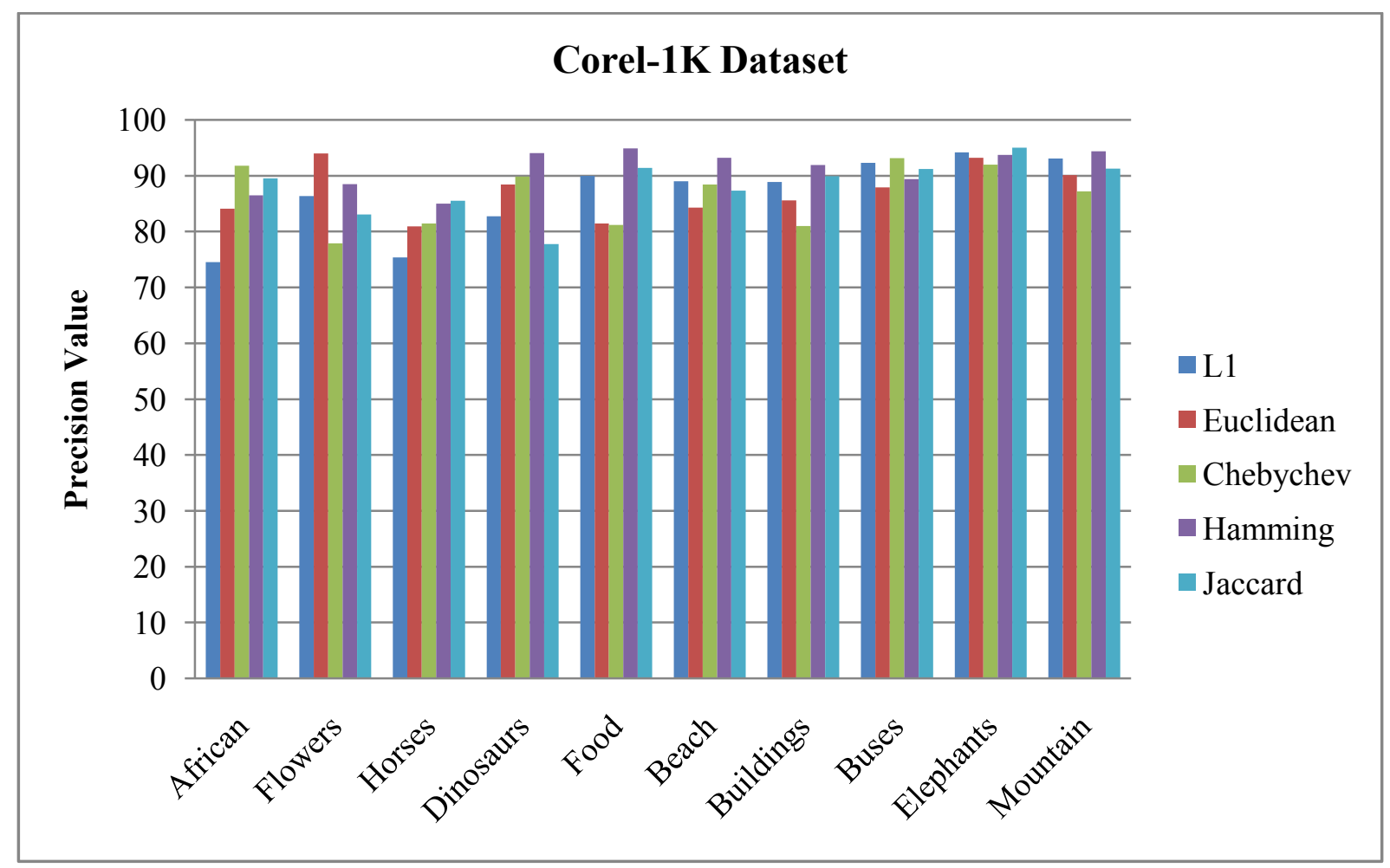

Fig. 9. The different similarity metrics based on precision value (L1, Euclidean, Chebychev, Hamming, Jaccard) on Corel-1K Dataset

- Corel-5k Dataset: This dataset consists of various classes of images and for each class, 5000 images are available. The sample image of the dataset is shown in Fig. 10. Accuracy, precision and recall are calculated to evaluate the performance of the proposed method. Similarity index of the image is calculated using L1, Euclidean, Chebychev, Hamming, Jaccard function and comparative analysis is shown in Table 4 and graph plotting is shown in Fig. 11. Sample results are shown in Fig. 12.

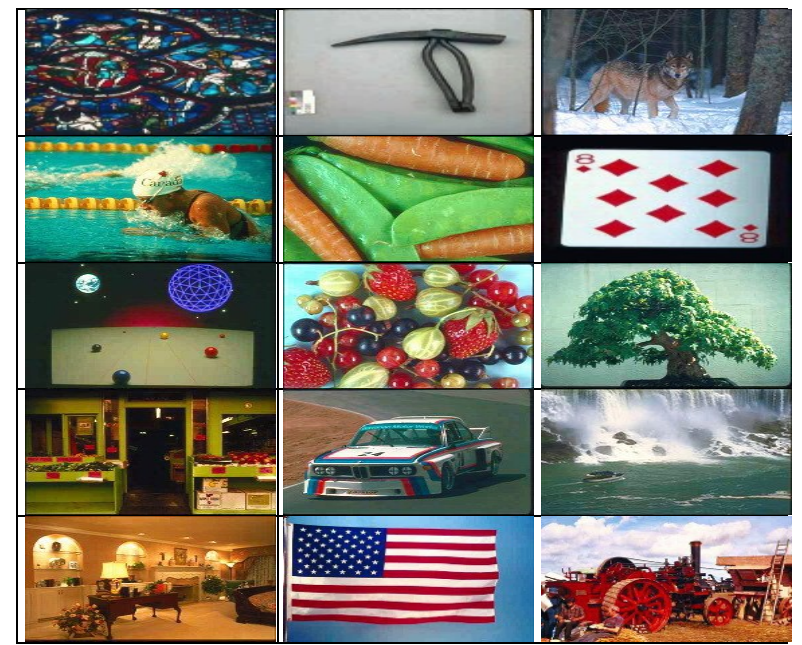

Fig. 10. Corel-5k (Few images from the dataset) 
Table 4: The different similarity metrics based on precision value (L1, Euclidean, Chebychev, Hamming, Jaccard) on Corel-5K Dataset

\begin{tabular}{|c|c|c|c|c|c|}
\hline & L1 & Euclidean & Chebychev & Hamming & Jaccard \\
\hline Lions & 69.81 & 70.46 & 67.58 & 64.43 & 60.65 \\
\hline Vegetables & 71.69 & 62.84 & 65.77 & 67.32 & 63.40 \\
\hline Weapons & 69.72 & 69.40 & 68.38 & 68.45 & 67.04 \\
\hline Churches & 71.39 & 70.35 & 70.86 & 69.32 & 68.07 \\
\hline Butterfly & 73.2 & 79.15 & 71.19 & 70.1 & 69.14 \\
\hline Bear & 75.61 & 85.22 & 79.91 & 71.5 & 71.2 \\
\hline Sunset & 74.03 & 84 & 78.81 & 72.3 & 72.9 \\
\hline Card & 73.69 & 86.16 & 74.93 & 75.6 & 77.48 \\
\hline Symbol & 70.15 & 79.15 & 71.4 & 77.9 & 76.5 \\
\hline Sippy & 78.2 & 77.99 & 82.5 & 79.9 & 79.1 \\
\hline People & 79.15 & 80.18 & 93.1 & 83.2 & 80.1 \\
\hline Cloud & 81.1 & 76.23 & 85.1 & 80.1 & 83.54 \\
\hline Beers & 82.3 & 76.33 & 74.3 & 79.1 & 83.1 \\
\hline Trees & 80.11 & 71.15 & 91.5 & 74.6 & 81.66 \\
\hline Doors & 79.4 & 74.56 & 78.9 & 80.25 & 76.4 \\
\hline Cars & 83.14 & 71 & 74.8 & 83.8 & 79.49 \\
\hline Bridges & 86.09 & 85.16 & 71.9 & 82.2 & 75.5 \\
\hline Waterfalls & 79.15 & 70.1 & 82.1 & 81.1 & 45.3 \\
\hline Bullock Cart & 76.4 & 76.49 & 69.9 & 80.1 & 78.3 \\
\hline Revolver & 77.89 & 72.23 & 73.1 & 79.8 & 74.3 \\
\hline Pattern & 79.11 & 77.17 & 74.5 & 78.4 & 69.9 \\
\hline Flag & 75.91 & 74.14 & 77.7 & 76.4 & 73.85 \\
\hline Art & 78.48 & 77.01 & 76.9 & 71.2 & 79.15 \\
\hline
\end{tabular}




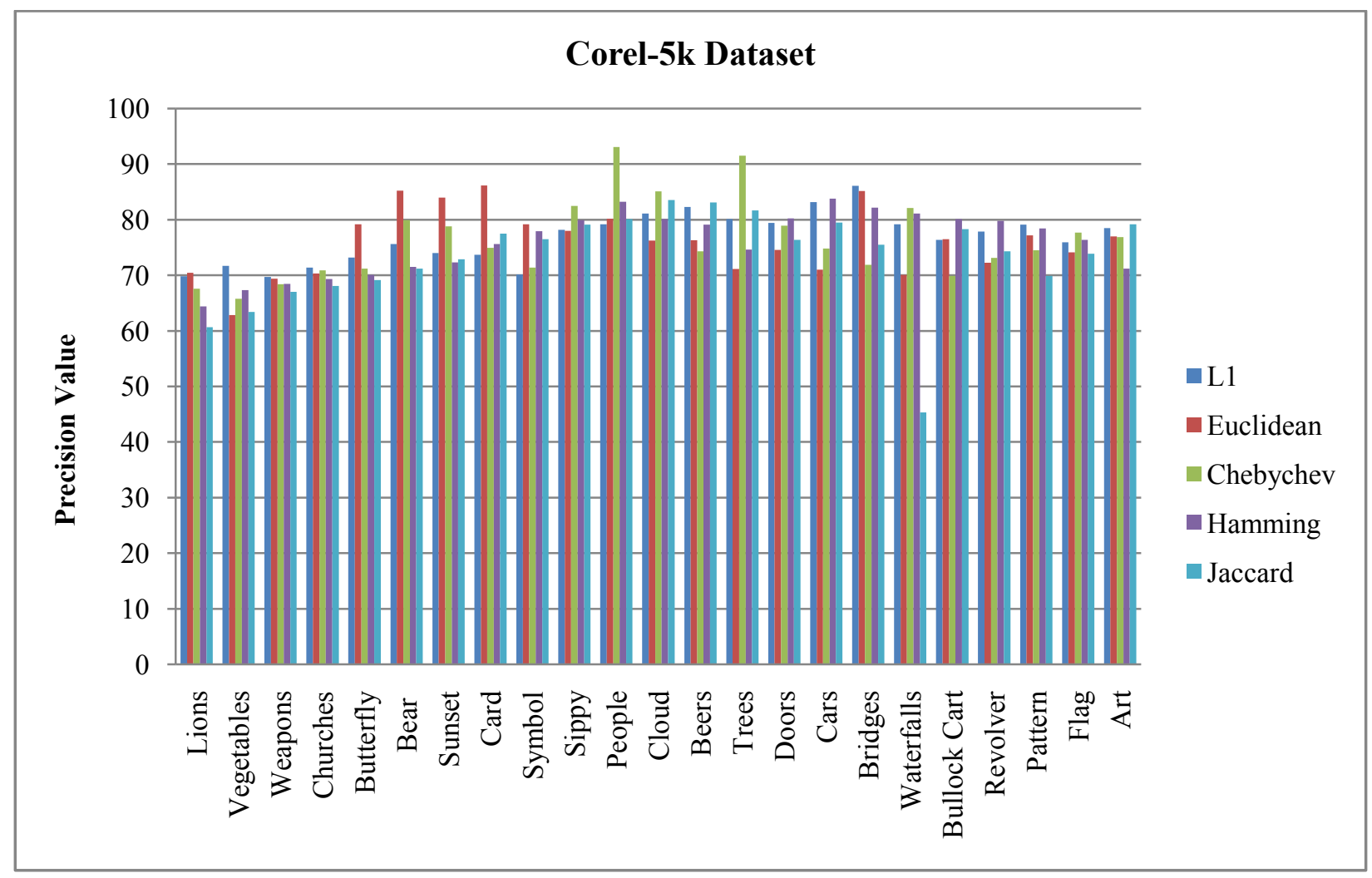

Fig. 11. The different similarity metrics based on precision value (L1, Euclidean, Chebychev, Hamming, Jaccard) on Corel-5K Dataset

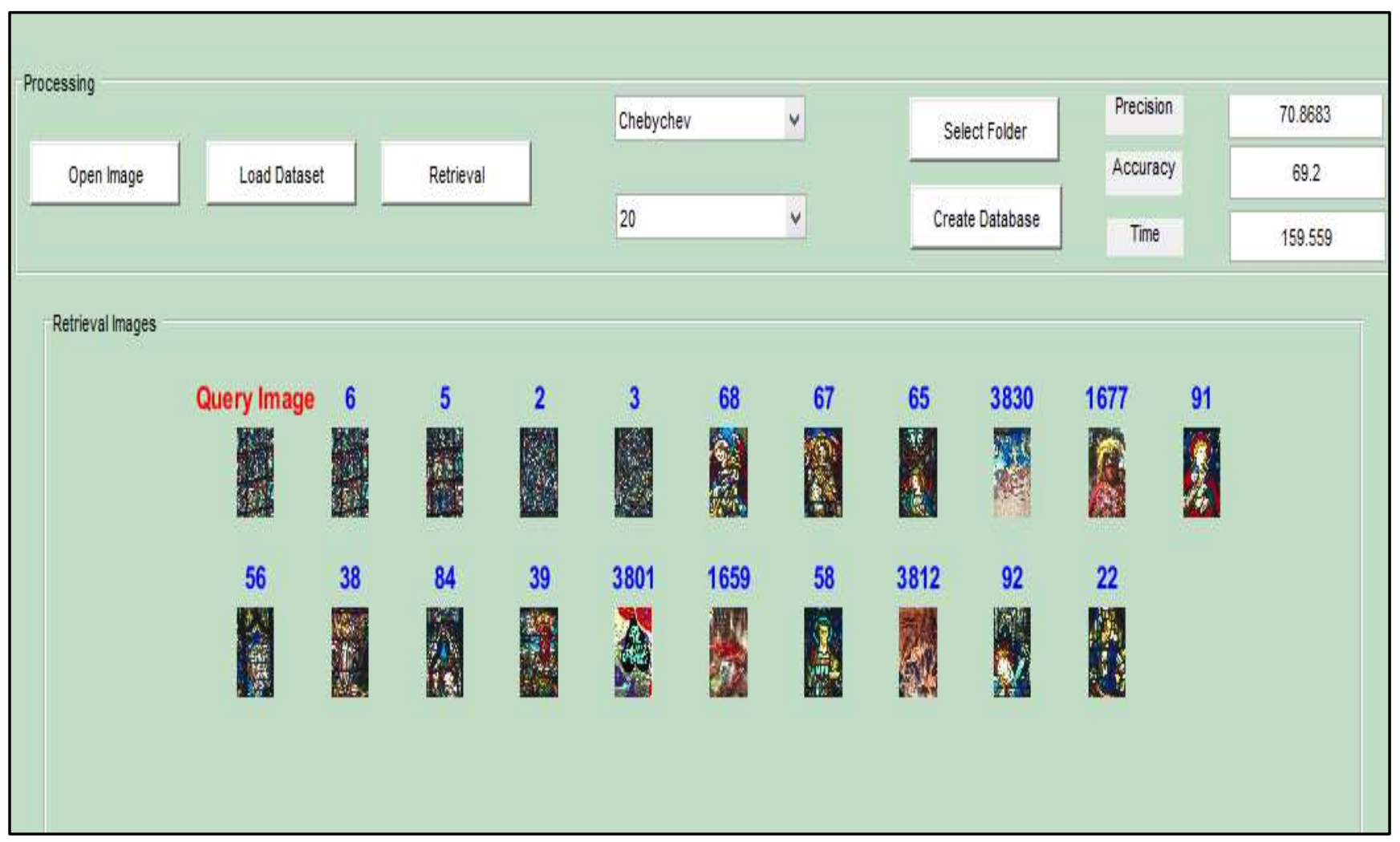

Fig. 12. Extractions of 20 Images for Churches(Corel-5k dataset). 


\subsection{Comparison Analysis of Proposed Work:}

After implementing the proposed methodology, the comparative analysis of the results has been done with various states of art methods. For the comparison of results, accuracy, precision and recall are used as parameters. The results of comparative analysis shown in Table 5, 7 and plotting of the graph are shown in Fig. 13-14. The proposed methodology takes less time for feature extraction; therefore, the proposed methodology is better than existing methods, as shown in Table 6. ROC curve is also identified during the evaluation process and the results are shown in Fig. 15.

Table 5: Comparision of an average value of accuracy, precision and recall with other states of the art method

\begin{tabular}{|c|c|c|c|c|c|c|}
\hline Dataset & $\begin{array}{c}\text { MTSD } \\
\text { Accuracy } \\
(\%)\end{array}$ & $\begin{array}{c}\text { Proposed } \\
\text { Accuracy } \\
(\%)\end{array}$ & $\begin{array}{c}\text { MTSD } \\
\text { Precision } \\
(\%)\end{array}$ & $\begin{array}{c}\text { Proposed } \\
\text { Precision } \\
(\%)\end{array}$ & $\begin{array}{c}\text { MTSD } \\
\text { Recall } \\
(\%)\end{array}$ & $\begin{array}{c}\text { Proposed } \\
\text { Recall } \\
(\%)\end{array}$ \\
\hline Corel-1k & 68.50 & $\mathbf{8 0 . 6 6}$ & 79.28 & $\mathbf{8 6 . 3 6}$ & 63.15 & $\mathbf{8 5 . 3 7}$ \\
\hline Corel-5k & 52.42 & $\mathbf{7 3 . 6}$ & 62.98 & $\mathbf{7 5 . 8 4}$ & 62.6 & $\mathbf{7 9 . 9 1}$ \\
\hline
\end{tabular}

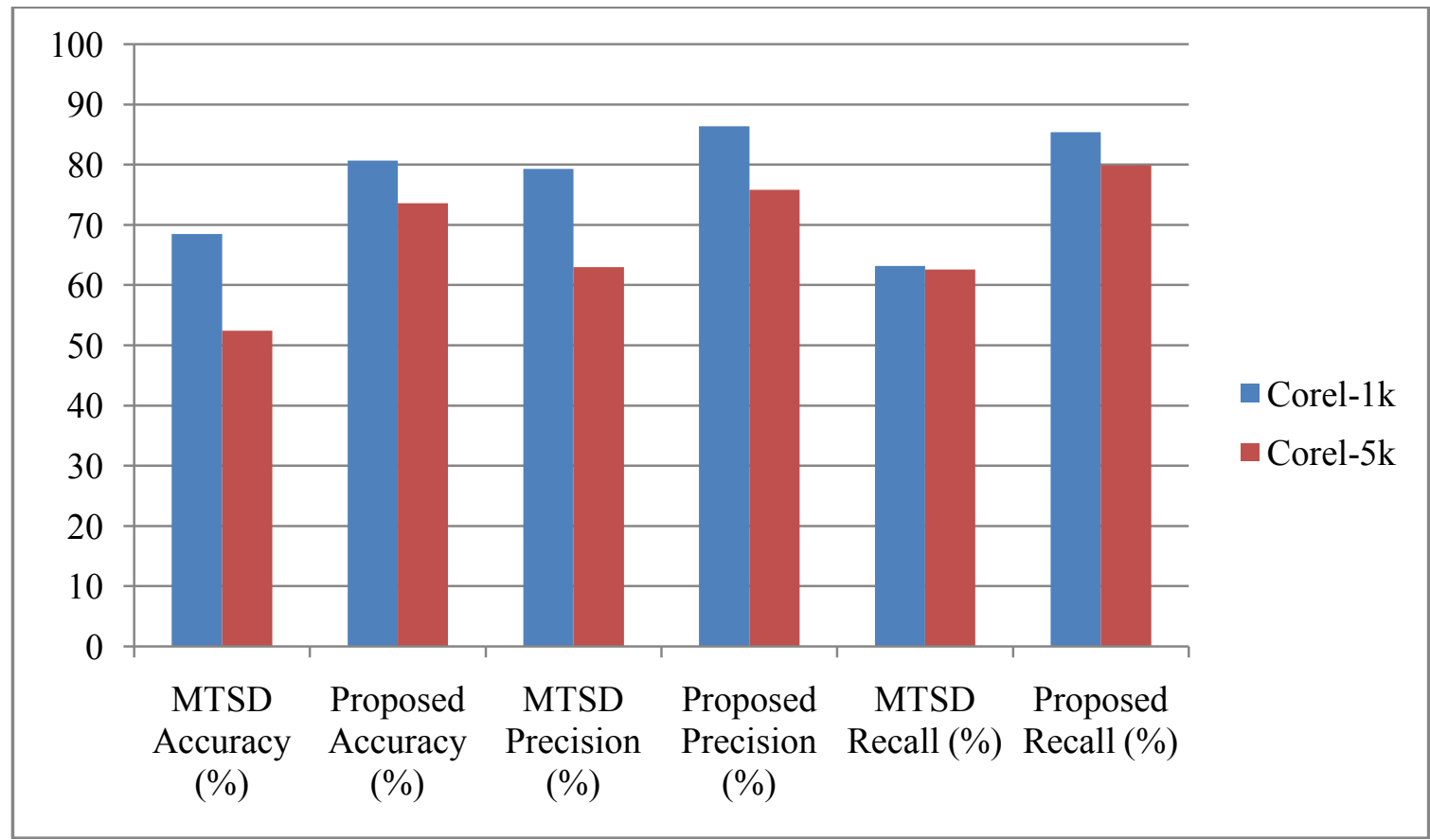

Fig. 13. Comparison of an average value of accuracy, precision and recall with another state of the art methods 
Table 6: Time Dimension of the proposed work

\begin{tabular}{|c|c|}
\hline Techniques & $\begin{array}{c}\text { Time Feature } \\
\text { Extraction (sec.) }\end{array}$ \\
\hline MTSD & 9.1615 \\
\hline Proposed Method & 6.7013 \\
\hline
\end{tabular}

Table 7: Comparison of average precision value with other states of the art methods

\begin{tabular}{|c|c|c|c|c|c|c|}
\hline Dataset & $\begin{array}{c}\text { Kaur [7] } \\
\text { Precision } \\
\mathbf{( \% )}\end{array}$ & $\begin{array}{c}\text { MTH [9] } \\
\text { Precision } \\
\mathbf{( \% )}\end{array}$ & $\begin{array}{c}\text { MSD 10] } \\
\text { Precision } \\
\mathbf{( \% )}\end{array}$ & $\begin{array}{c}\text { Varish [8] } \\
\text { Precision } \\
\mathbf{( \% )}\end{array}$ & $\begin{array}{c}\text { MTSD [6] } \\
\text { Precision } \\
(\%)\end{array}$ & $\begin{array}{c}\text { Proposed } \\
\text { Precision } \\
\mathbf{( \% )}\end{array}$ \\
\hline $\begin{array}{c}\text { Corel- } \\
\text { Dataset-1k }\end{array}$ & 73.4 & 69.32 & 74.67 & 60.2 & 79.28 & $\mathbf{8 6 . 3 6}$ \\
\hline $\begin{array}{c}\text { Corel- } \\
\text { Dataset-5k }\end{array}$ & - & 51.84 & 57.92 & - & 62.98 & $\mathbf{7 1 . 3 8}$ \\
\hline
\end{tabular}

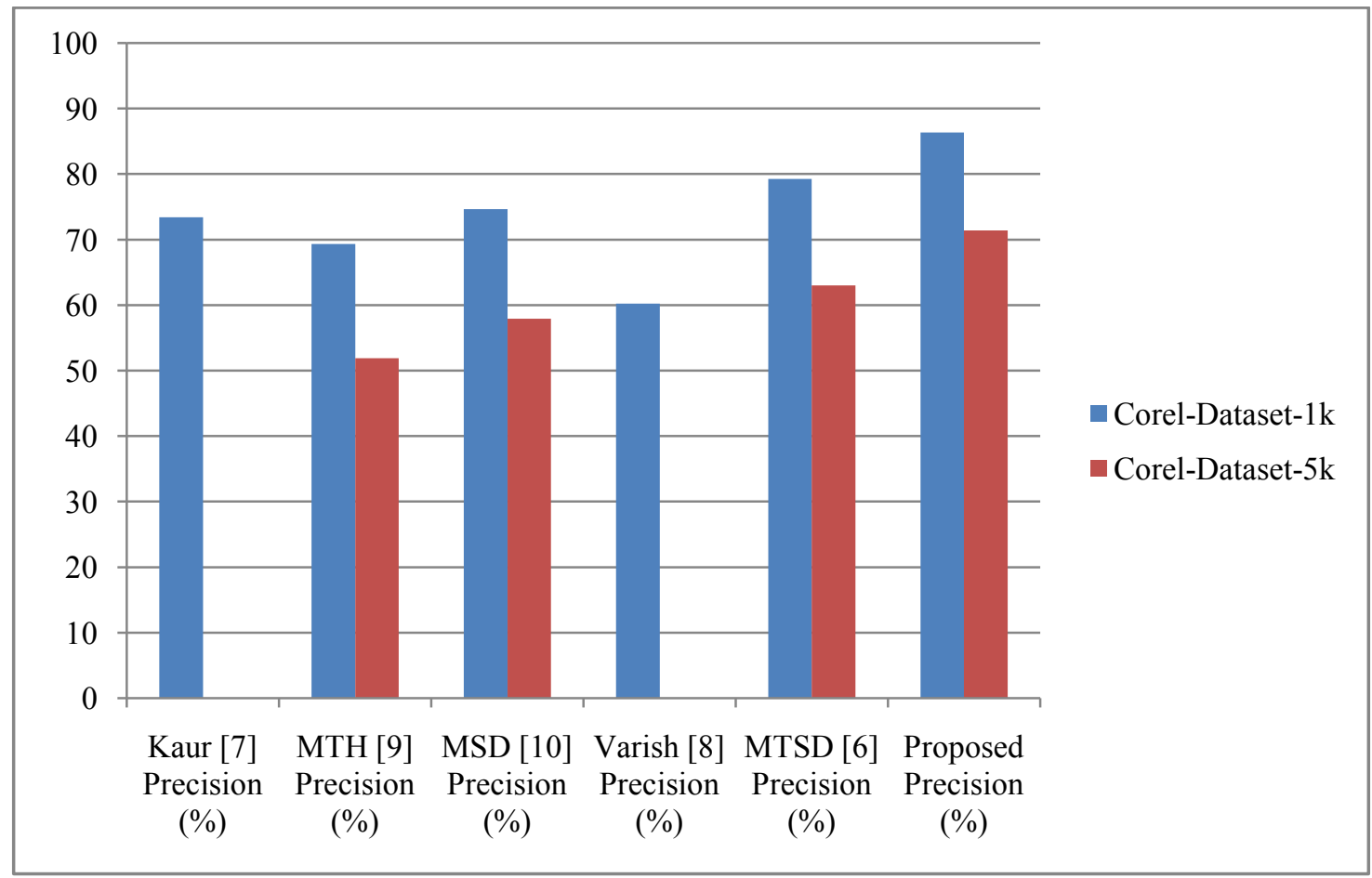

Fig. 14. Extractions of 20 Images for Churches(Corel-5k dataset). 


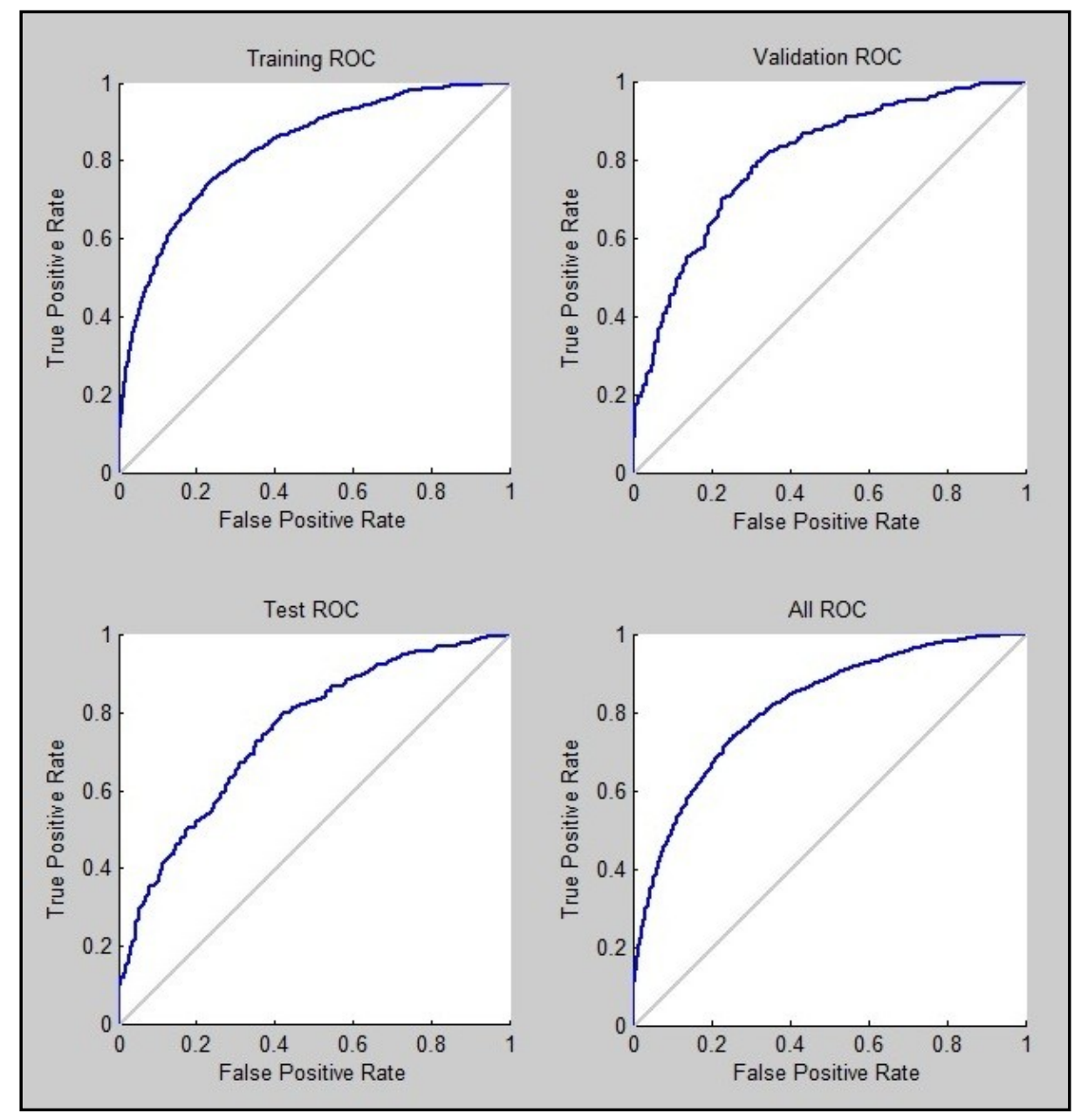

Fig. 15. ROC curves for all steps while evaluating on the standard database.

\section{Conclusion}

CBIR is a mining image technique used to retrieve the desired image from an extensive image database. The proposed algorithm combines the characteristics of texture, shape and color. This work implements a new scheme of enhanced CBIR by utilizing multi-feature as well as neural networks with color points. Color features of image stem by DCD and DCD is the quantization of color either in RGB HSV field and value with the histogram of any image. The extensive presentation of these schemes based on the accuracy it gives. Thus, the research performed on different image databases and the projected CBIR scheme with previous related work exposed projected work consequences in privileged accuracy in expressions of exactitude and correctness rate, i.e., $87 \%$ for the Corel-1k and $97 \%$ for Corel-5k datasets. The results of the proposed method are better than existing image retrieval methods. This method could be helpful for other computer vision-related applications. In the future, this technique can be applied for face and skin detection to increase the versatility of the proposed method. 


\section{Funding Information: Funding information is not applicable / No funding was received.}

Conflict of Interest: Dr. Sandeep Kumar is currently working as a Dean R\&D and Professor in ECE department of Sreyas Institute of Engineering and Technology, Hyderabad, India. Dr. Sandeep Kumar declares that he has no conflict of interest. Dr. Rohit Raja is presently working as an Associate professor at Central University, Chhattisgarh, India. Dr. Rohit Raja declares that he has no conflict of interest. Prof. Shipa Rani is currently working as an Assistant Professor at Neil Gogte Engineering and Technology, India. Prof. Shipa Rani states that she has no conflict of interest. Prof. Hemlata is currently working as an Assistant Professor at Sreyas Institute of Engineering and Technology, India. Prof. Hemlata states that she has no conflict of interest

Ethical approval: All procedures performed in studies involving human participants followed the institutional or national research committee's ethical standards.

\section{References}

[1] Dr. H. B. Kekre, Priyadarshini Mukherjee, Shobhit Wadhwa," Image Retrieval with shape features extracted using gradient operators and slope magnitude method with BTC," International Journal of Computer Applications (0975 - 8887), Volume 6- No.8, September 2010 .

[2] Raman Maini \& Dr. Himanshu Aggarwal, "Study and Comparison of Various Image Edge Detection Techniques," International Journal of Image Processing (IJIP), Volume (3), issue 1, 2009.

[3] Sudeep D. Thepade, Yogita D. Shinde "Improvisation of Content-Based Image Retrieval using Color Edge Detection with various Gradient Filters and Slope Magnitude Method" International Conference on Computing Communication Control and Automation, IEEE-2015.

[4] Dr. H. B. Kekre, Sudeep D. Thepade, Varun K. Banura, "Image Retrieval using Shape Texture Patterns generated from Walsh-Hadamard Transform and Gradient Image" International Journal of Computer Science and Information Security 01/2010.

[5] Dr.H.B.Kekre, Sudeep D. Thepade, "Image Retrieval using Augmented Block Truncation Coding," International Conference on Advances in Computing, Communication and Control (ICAC3'09), Mumbai, Maharashtra, India, pp 384-390, January 23-24, 2009.

[6] N. Dalal and B. Triggs, Histograms of oriented gradients for human detection, in Computer Vision and Pattern Recognition, 2005. CVPR 2005. IEEE Computer Society Conference on, vol. 1. IEEE, 2005, pp.886-893.

[7] H. Shao, Y. Wu, W. Cui, J. Zhang, "Image Retrieval Based on MPEG-7 Dominant Color Descriptor," The 9th International Conference for Young Computer Scientists, pp. 753-757, 2008.

[8] Peng Wu, Yong Man Ro, Chee Sun Won, and Yang Lim Choi," Texture Descriptors in MPEG7," Computer Analysis of Images and Patterns, vol. 2124, pp 21-28, August 2001

[9] Dong Kwon Park, Yoon Seok Jeon, and Chee Sun Won, " Efficient use of Local Edge Histogram Descriptor," ACM Workshop on multimedia, November 2000, pp. 51-55

[10] Pranoti Mane, and N. G. Bawane," Comparative Performance Evaluation of Edge Histogram Descriptors and Color Structure Descriptors in Content-Based Image Retrieval," National Conference on Innovative Paradigms in Engineering \& Technology (NCIPET-2015

[11] C.S. Won, D.K. Park, and S.J. Park," Efficient use of Edge Histogram Descriptor-An Overview,” ETRI Journal, vol. 24, Number 1, Feb 2002, pp. 23-30. 
[12] Sawat Somnugpong, and Kanokwan Khiewwan," Content-based image retrieval using a combination of color correlograms and edge direction histogram," 978-1-5050-20331/16/C2016 IEEE

[13] Nipa Chowdhury and Mohammad Abul kashem "A Comparative Analysis of Feed-forward Neural Network \& Recurrent Neural Network to Detect Intrusion" 5th International Conference on Electrical and Computer Engineering ICECE, IEEE- 2008.

[14] Christos Stergiou and Dimitrios Siganos, Neural networks.

[15] Yati Dandotiya and Anshul Atre "Image Retrieval using Edge Detection, RLBP, Color Moment Method for YCbCr and HSV Color Space" International Conference on Electronics, Communication and Aerospace Technology, IEEE-2017.

[16] Swati Agarwal, A. K. Verma and Nitin Dixit "Content-Based Image Retrieval using Color Edge Detection and Discrete Wavelet Transform" IEEE-2014.

[17] Srikanth Redrouthu, Annapurani. K "Time Comparison of Various Feature Extraction of Content-Based Image Retrieval" International Journal of Computer Science and Information Technologies, Vol. 5 (2), PP. 2518-2523, 2014.

[18] Atif Nazir, Rehan Ashraf, Taiha Hamdani and Nouman Ali "Content-Based Image Retrieval System " using HSV Color Histogram, Discrete Wavelet Transform and Edge Histogram Descriptor" 8 International Conference on Computing, Mathematics and Engineering Technologies - iCoMET 2018.

[19] Yubing Dong, Mingjing Li and Jie Li "Image Retrieval Based on Improved Canny Edge Detection Algorithm" International Conference on Mechatronic Sciences, Electric Engineering and Computer (MEC) Dec 20-22, 2013.

[20] Raja, R., Kumar, S. \& Mahmood, M.R. Color Object Detection Based Image Retrieval Using ROI Segmentation with Multi-Feature Method. Wireless Pers Commun (2020). https://doi.org/10.1007/s11277-019-07021-6.

[21] Rohit Raja; Tilendra Shishir Sinha; Raj Kumar Patra; Shrikant Tiwari Physiological trait-based biometrical authentication of human-face using LGXPandANNtechniques by International Journal of Information and Computer Security (IJICS), Vol. 10, No. 2/3, 2018.

[22] Rani S., Lakhwani K., Kumar S. (2021) Three Dimensional Wireframe Model of Medical and Complex Images Using Cellular Logic Array Processing Techniques. In: Abraham A. et al. (eds) Proceedings of the 12th International Conference on Soft Computing and Pattern Recognition (SoCPaR 2020). SoCPaR 2020. Advances in Intelligent Systems and Computing, vol 1383. Springer, Cham.

[23] Kumar S, Sukhwinder Singh, and Jagdish Kumar. "Gender Classification Using Machine Learning with Multi-Feature Method." In IEEE 9th Annual Computing and Communication Workshop and Conference (CCWC), pp. 0648-0653, Las Vegas, USA, January 7th-9th, 2019.

[24] Kumar S, Sukhwinder Singh, and Jagdish Kumar. "Multiple Face Detection Using Hybrid Features with SVM Classifier." In Data and Communication Networks, pp. 253-265. Springer, Singapore, 2019 with ISBN: 978-981-13-2254-9

[25] Kumar S, Sukhwinder Singh and Jagdish Kumar "Face Spoofing Detection Using Improved SegNet Architecture with Blur Estimation Technique" in International Journal of Biometric, Inderscience Publication. 


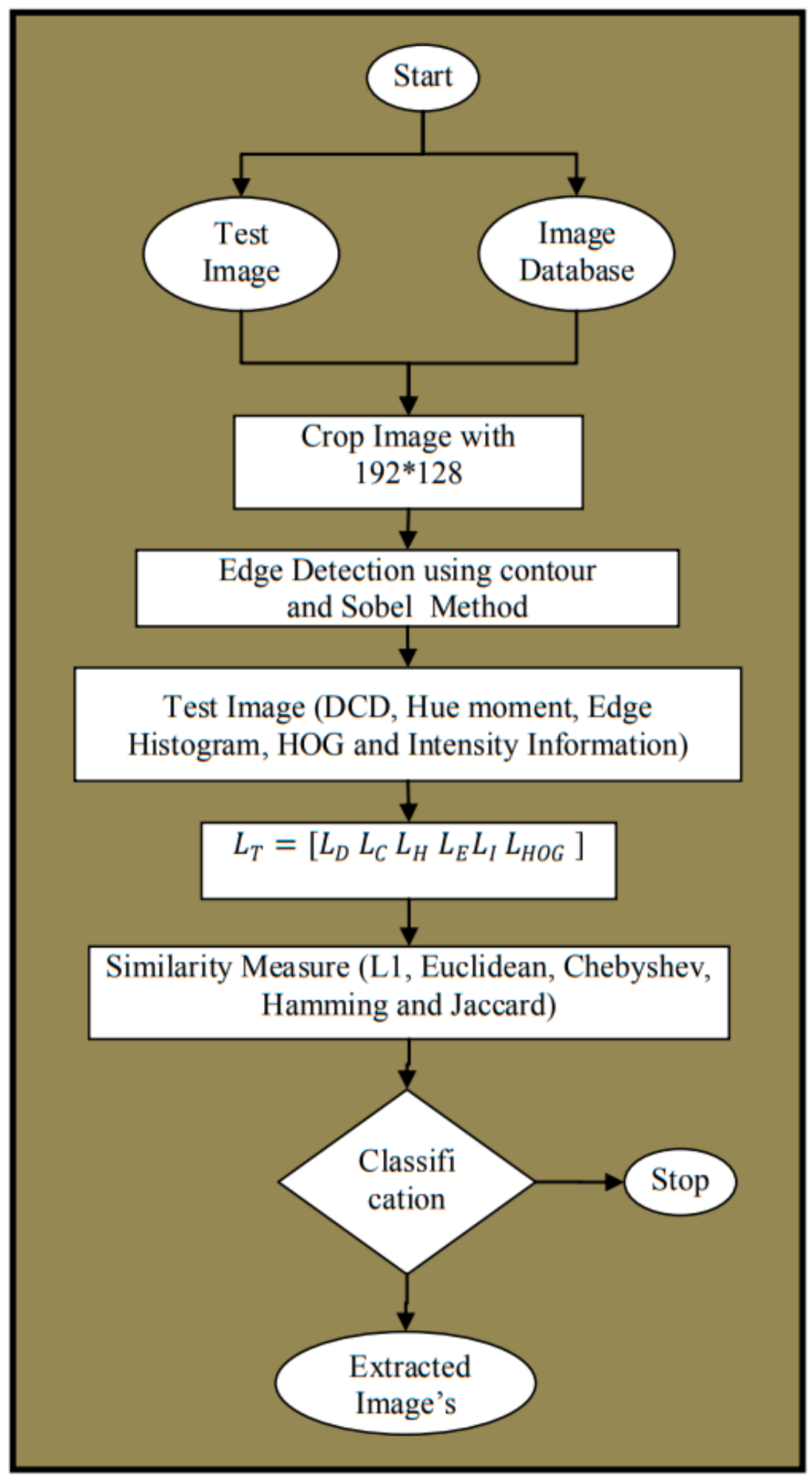

\section{Figure 1}

Flow Chart of Proposed Work 


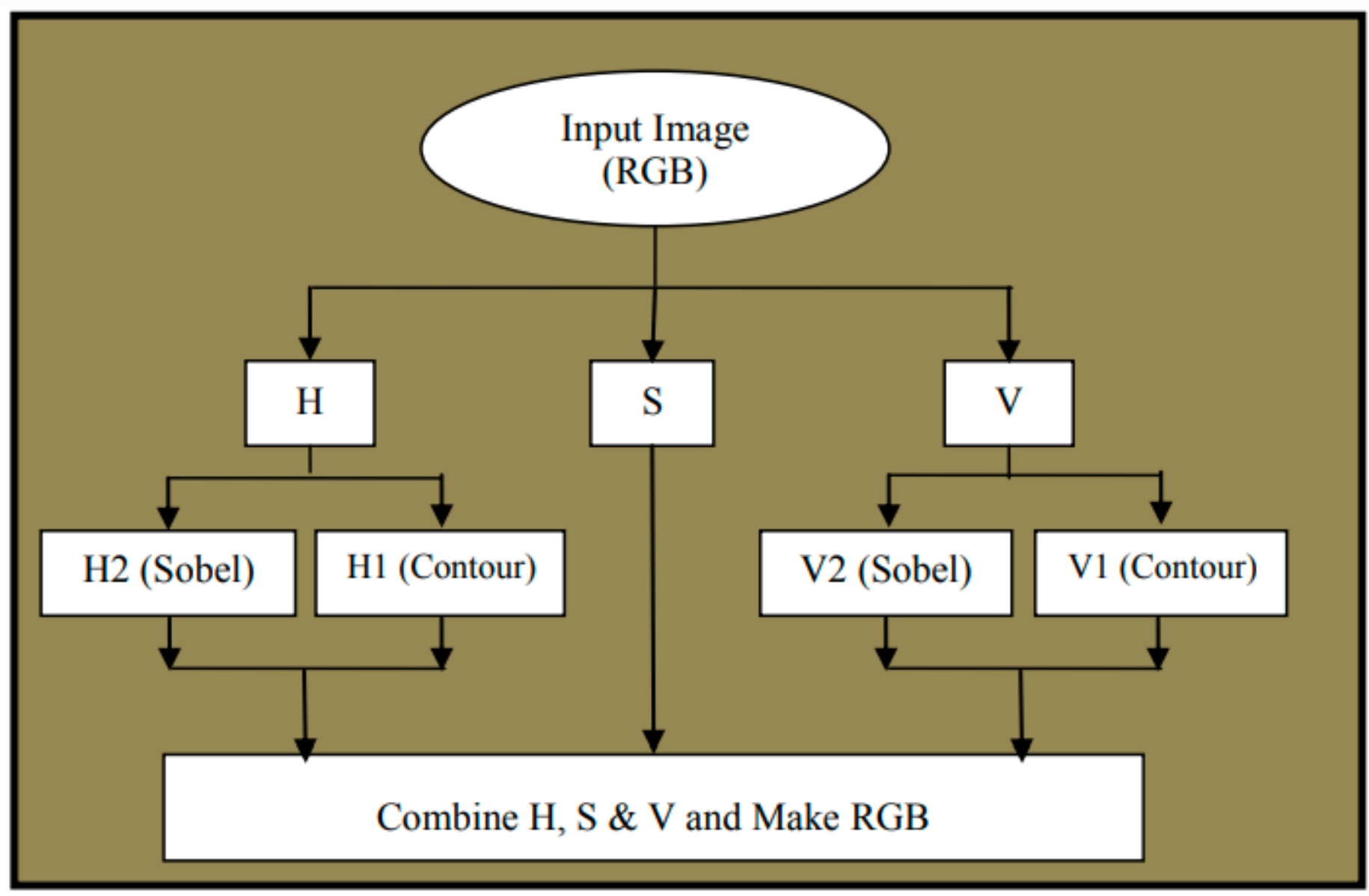

Figure 2

Flow Chart of edge detection of the proposed method.

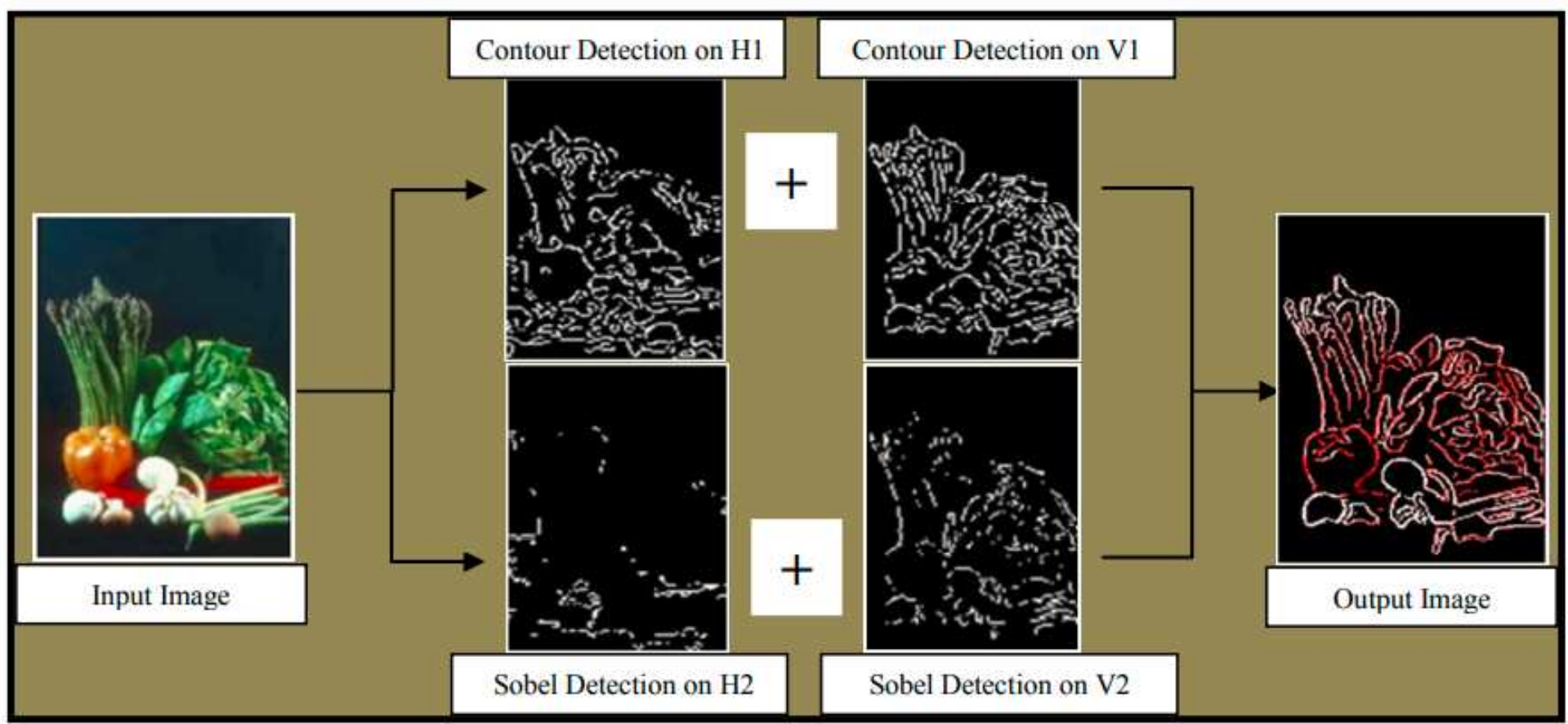

Figure 3 
Results on Vegitables Image.

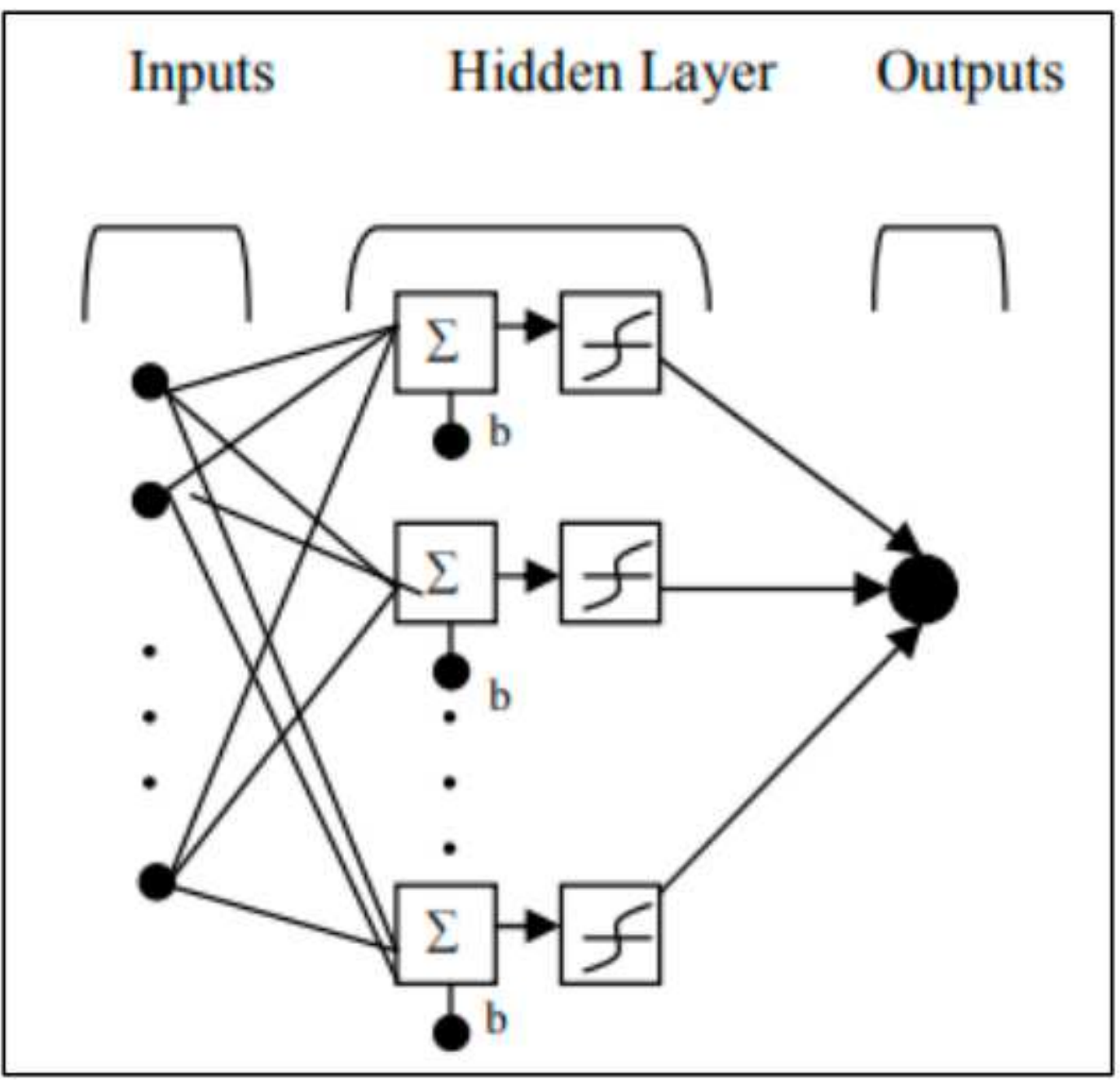

Figure 4

FEED-Forward Network 


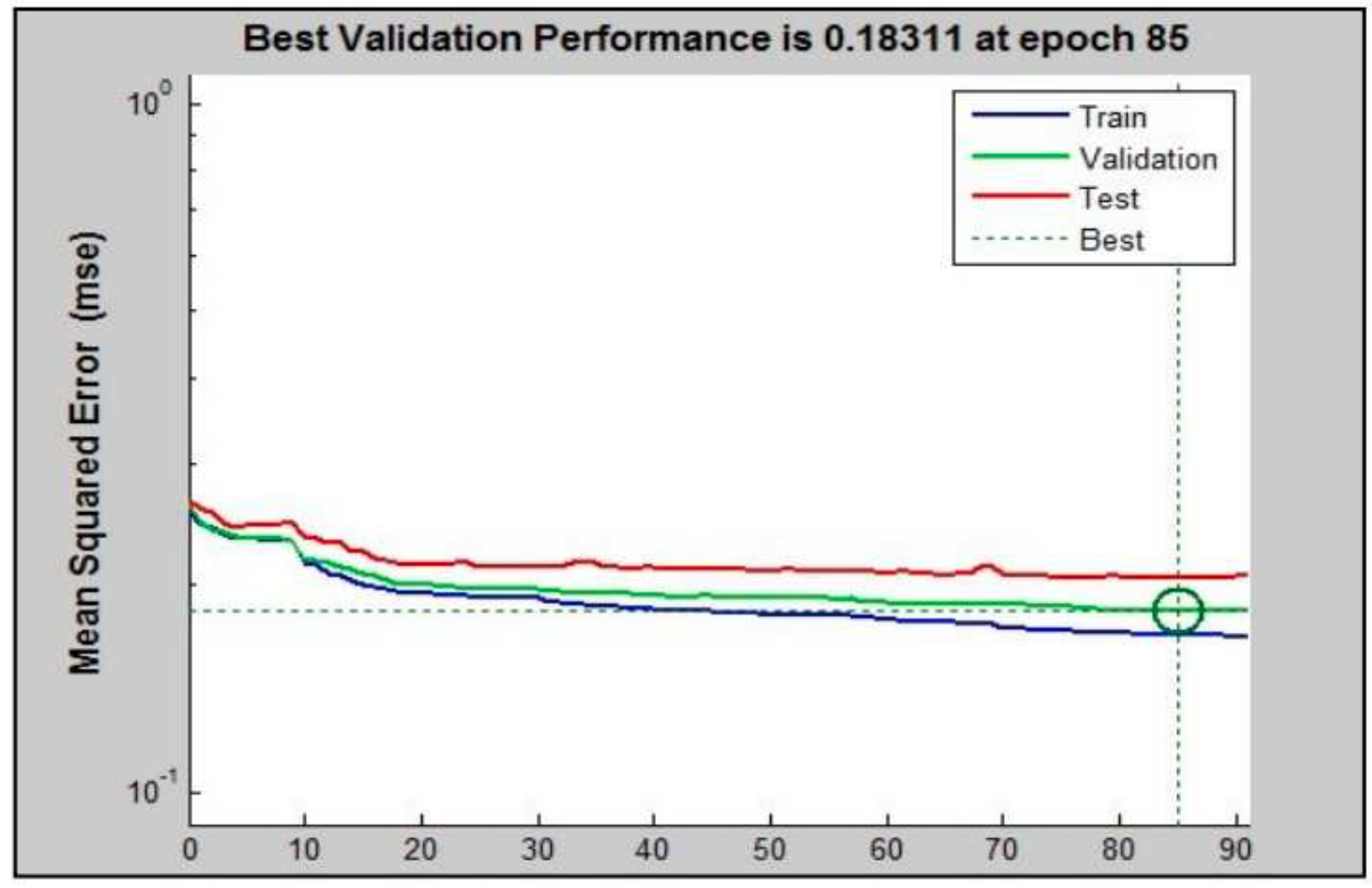

Figure 5

Validation performance sample 


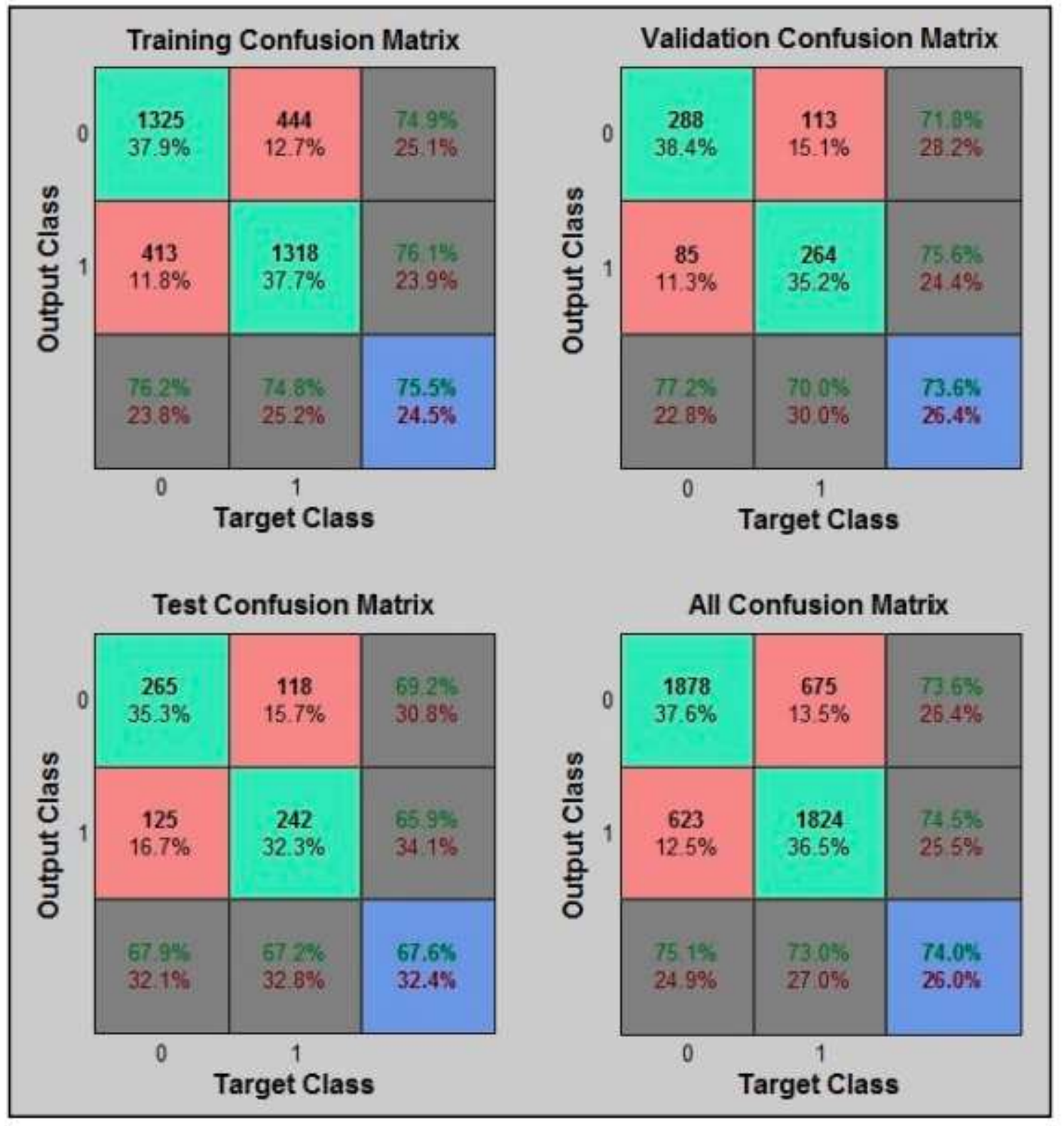

Figure 6

Overall confusion matrix on benchmark datasets 


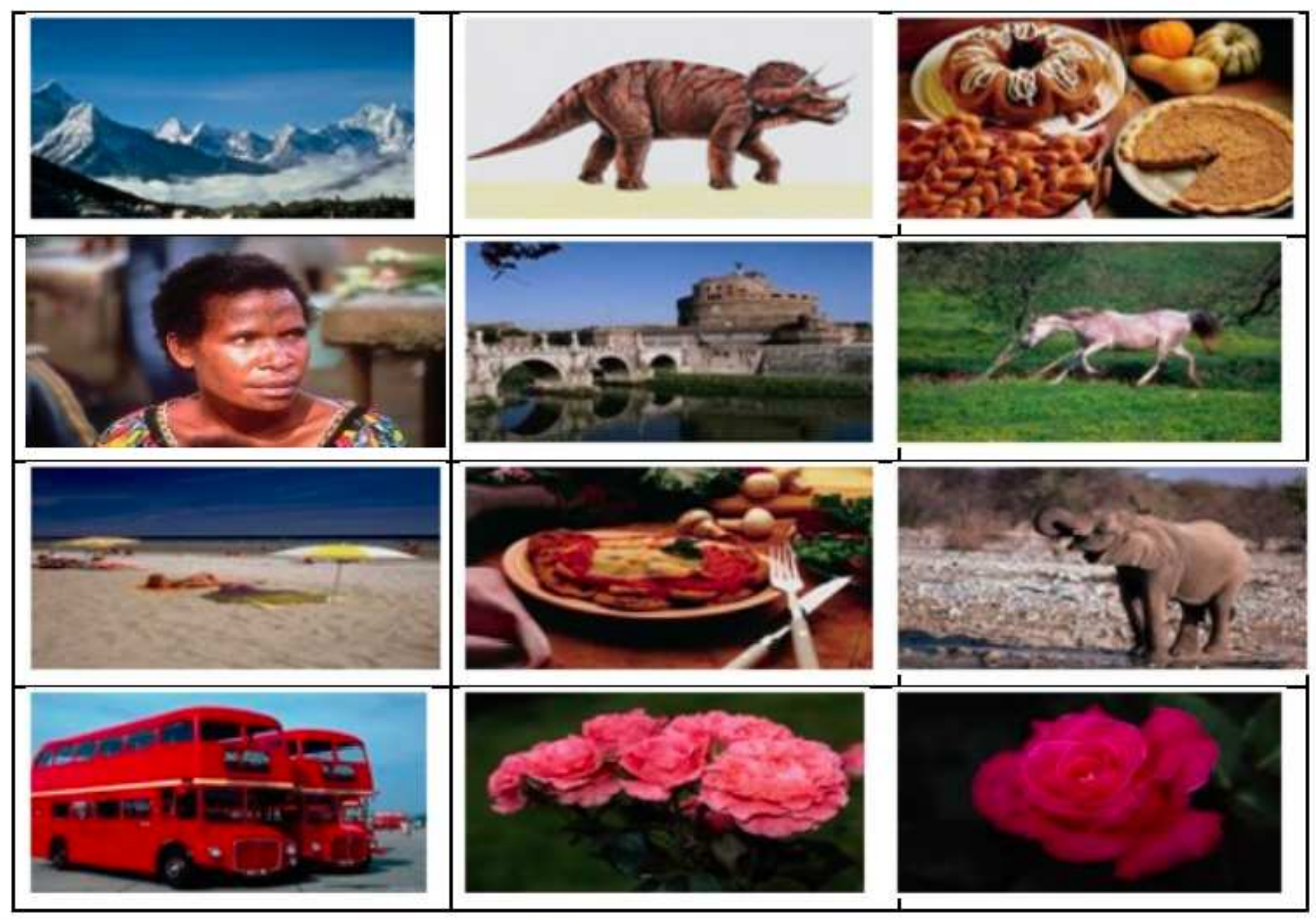

Figure 7

Corel-1k (Few images from the dataset) 


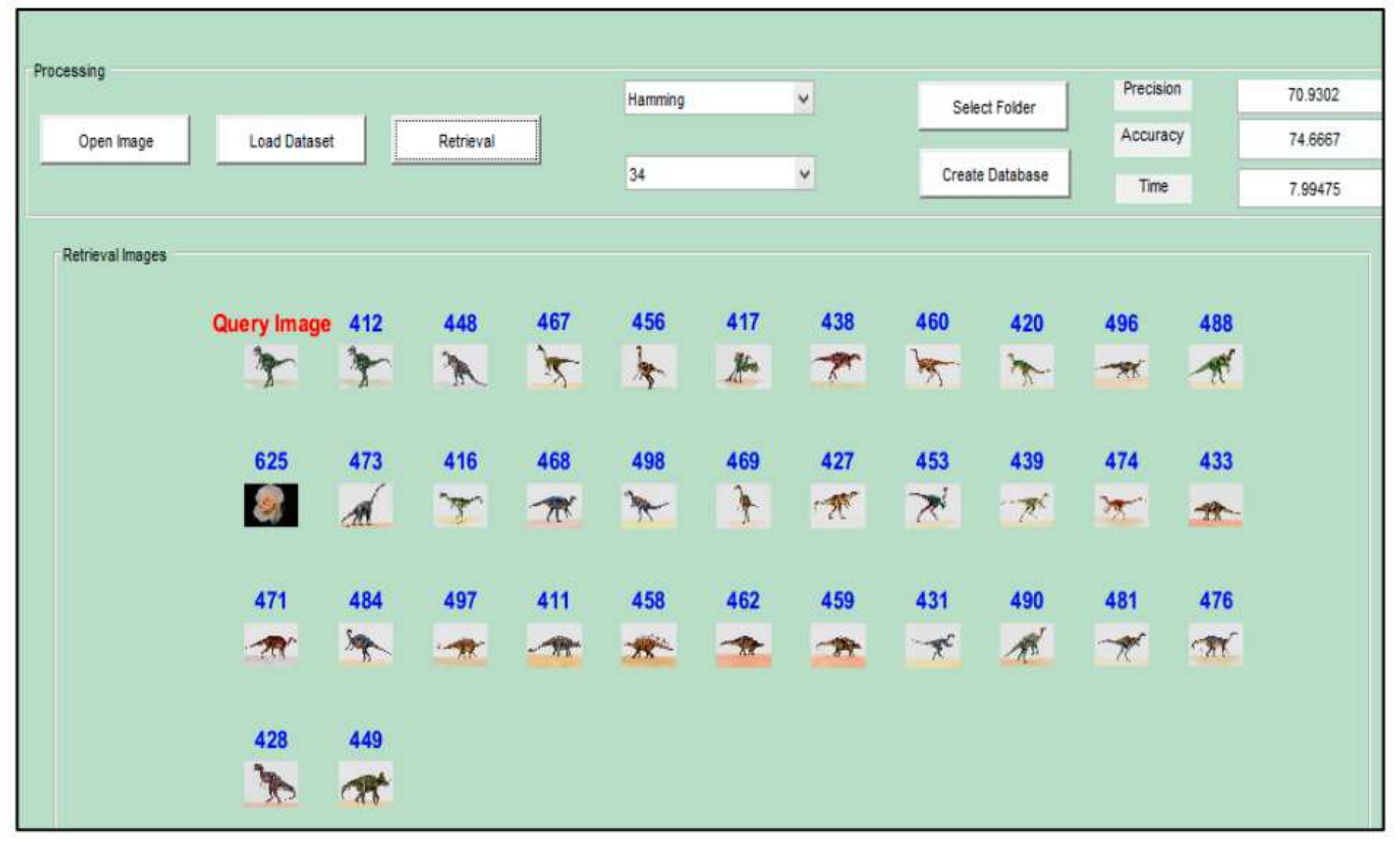

Figure 8

Dinosaur images are extracted from the dataset against 1 test/query image (Corel-1k dataset). 


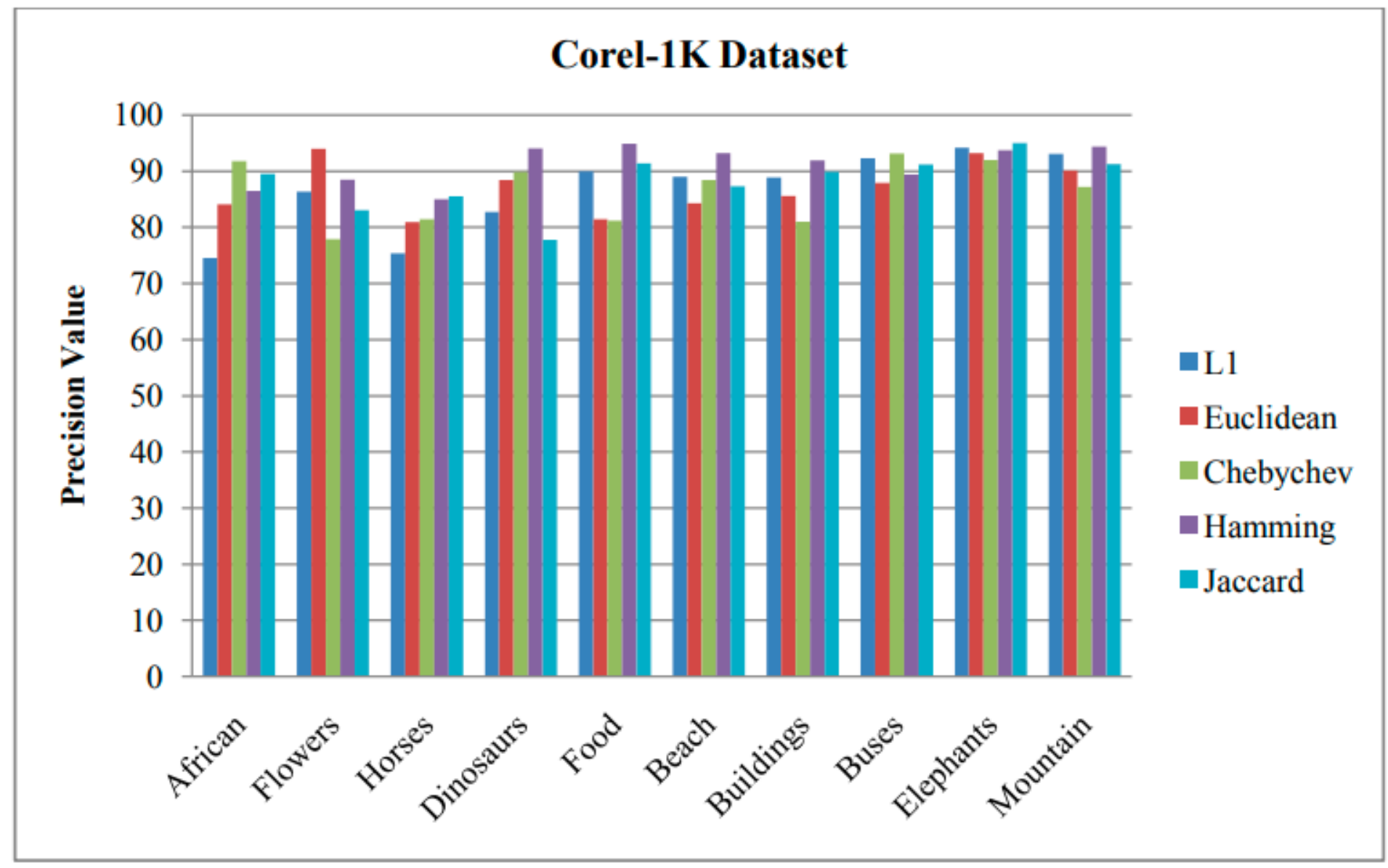

Figure 9

The different similarity metrics based on precision value (L1, Euclidean, Chebychev, Hamming, Jaccard) on Corel-1K Dataset 


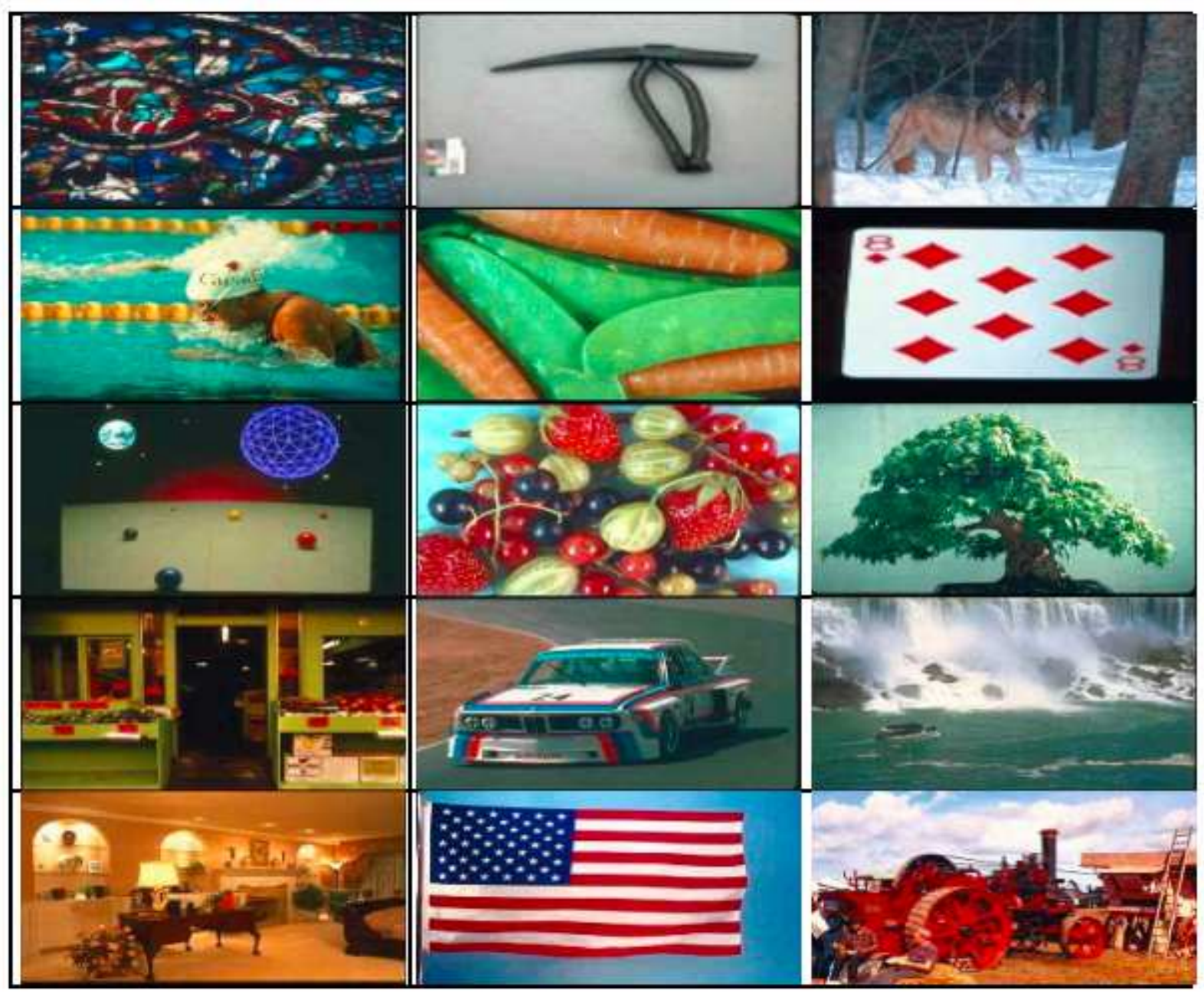

Figure 10

Corel-5k (Few images from the dataset) 


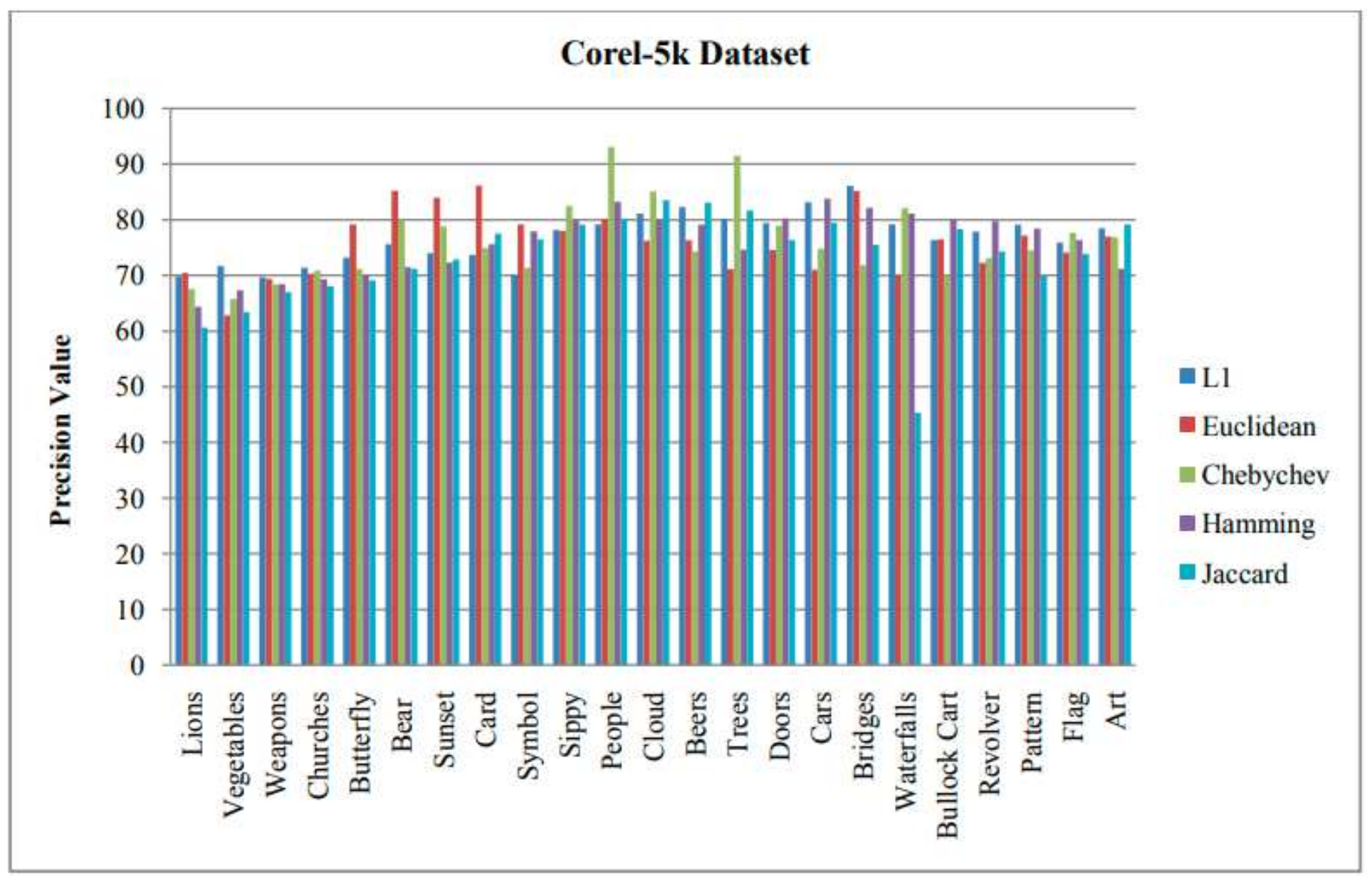

Figure 11

The different similarity metrics based on precision value (L1, Euclidean, Chebychev, Hamming, Jaccard) on Corel-5K Dataset 


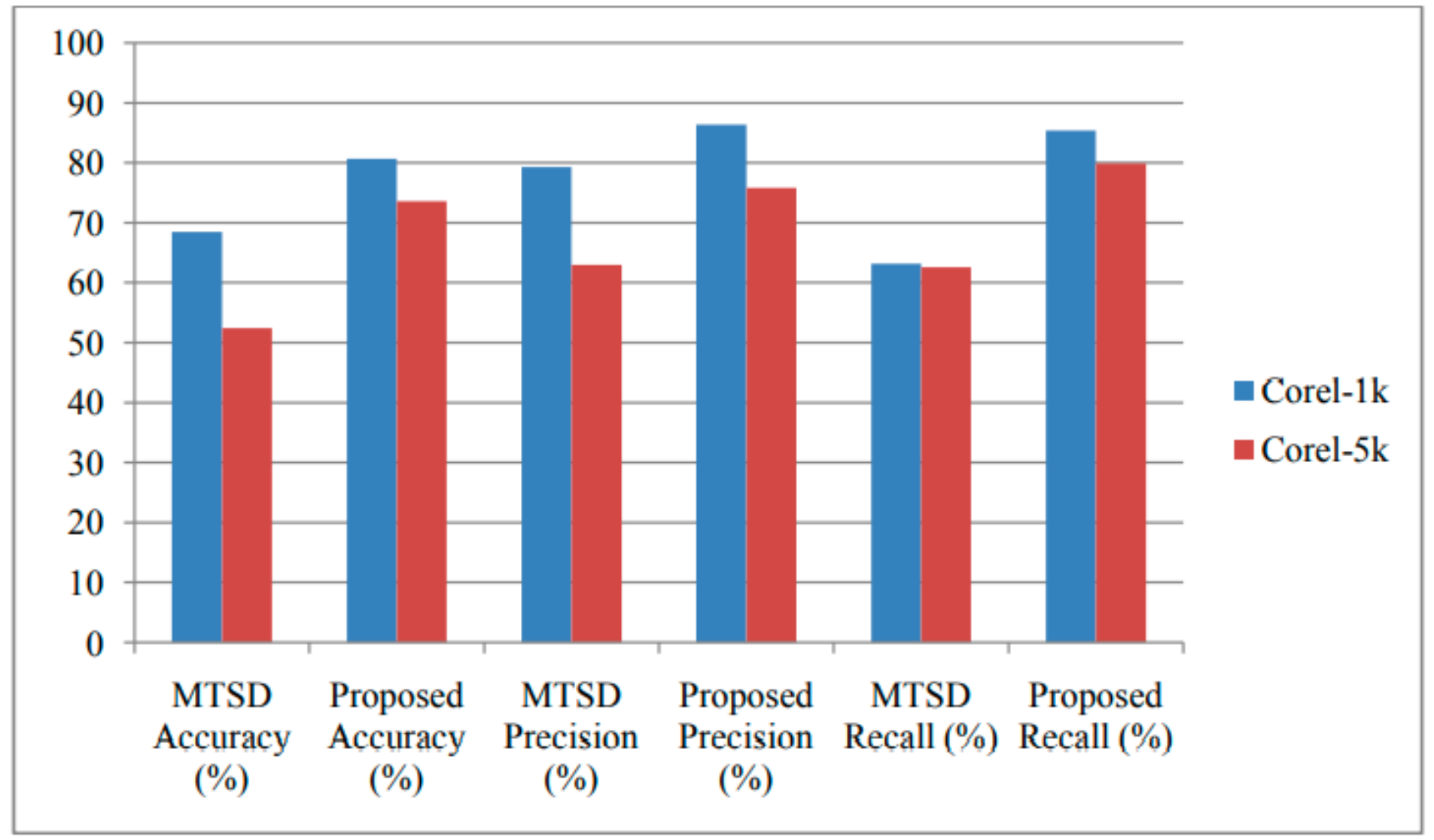

Figure 13

Comparison of an average value of accuracy, precision and recall with another state of the art methods 


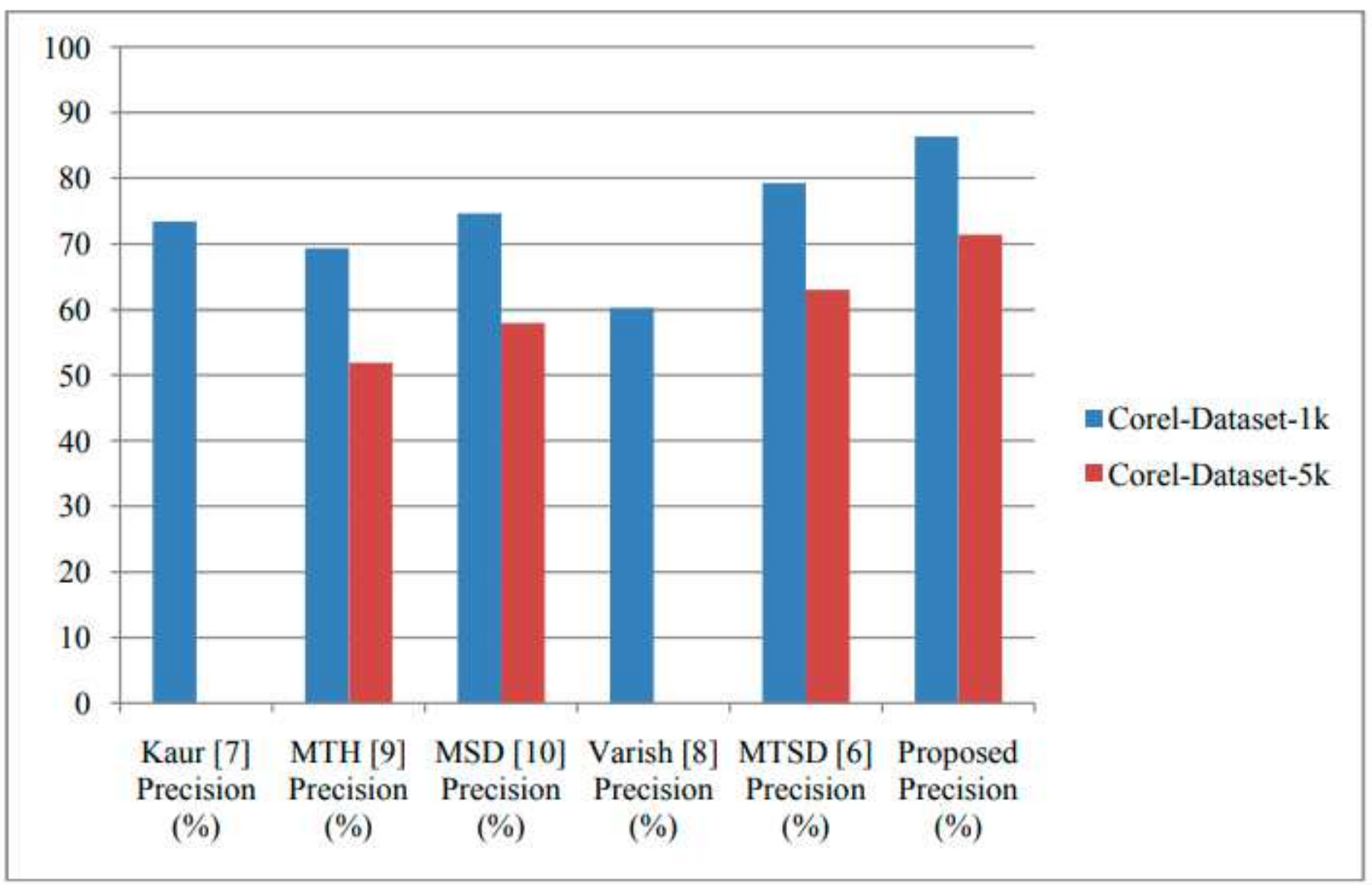

Figure 14

Extractions of 20 Images for Churches(Corel-5k dataset). 


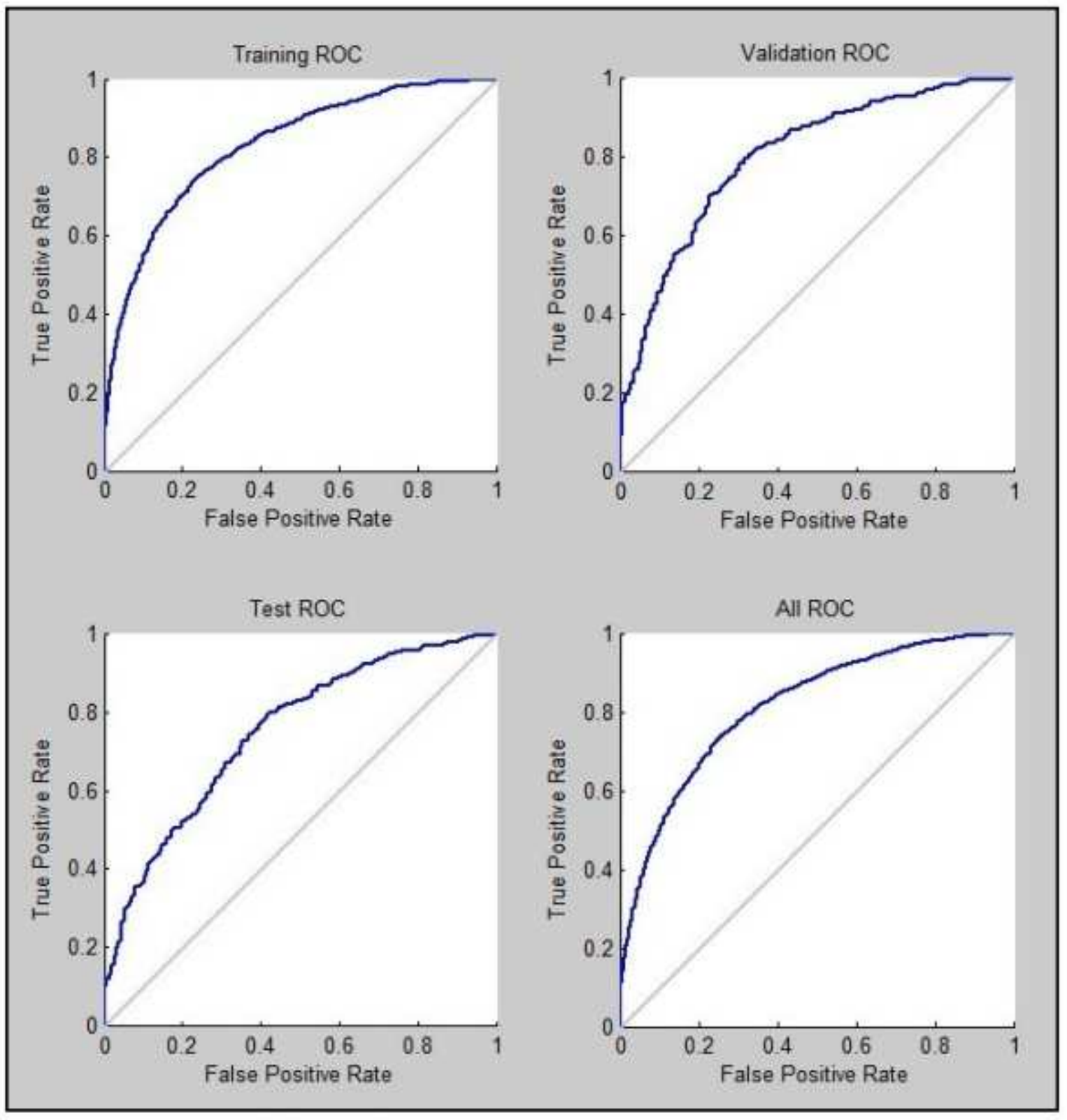

Figure 15

curves for all steps while evaluating on the standard database. 